\title{
Abnormal Auditory Experience Induces Frequency-Specific Adjustments in Unit Tuning for Binaural Localization Cues in the Optic Tectum of Juvenile Owls
}

\author{
Joshua I. Gold and Eric I. Knudsen \\ Department of Neurobiology, Stanford University, Stanford, California 94305-5125
}

Early auditory experience shapes the auditory spatial tuning of neurons in the barn owl's optic tectum in a frequencydependent manner. We examined the basis for this adaptive plasticity in terms of changes in tuning for frequency-specific interaural time differences (ITDs) and level differences (ILDs), the dominant sound localization cues. We characterized broadband and narrowband ITD and ILD tuning in normal owls and in owls raised with an acoustic filtering device in one ear that caused frequency-dependent changes in sound timing and level. In normal owls, units were tuned to frequency-specific ITD and ILD values that matched those produced by sound sources located in their visual receptive fields. In contrast, in device-reared owls, ITD tuning at most sites was shifted from normal by $\sim 55 \mu$ sec toward open-ear leading for $4 \mathrm{kHz}$ stimuli and $15 \mu \mathrm{sec}$ toward the opposite-ear leading for $8 \mathrm{kHz}$ stimuli,

The response properties of sensory neurons in the CNS can be extremely complex and, in some cases, reflect the experience of the individual. For example, visual neurons in the primate temporal cortex that respond preferentially to faces (Rolls et al., 1989; Perrett et al., 1992; Young and Yamane, 1992) and auditory neurons in the songbird forebrain that respond primarily to birdsong (Margoliash, 1983, 1986; Doupe and Konishi, 1991; Margoliash and Fortune, 1992) often respond best to stimuli that are familiar to the individual. The complex response properties of these neurons arise from their tuning to sets of spatial and temporal cues. In an analogous fashion, the spatial tuning of auditory neurons in the barn owl's optic tectum and its mammalian homolog, the superior colliculus, arises from their tuning to sets of sound localization cues (Wise and Irvine, 1985; Olsen et al., 1989; Brainard and Knudsen, 1993). In this study, we examined the degree to which this tuning matches the details of the individual's early experience.

Sound localization cues arise from interactions between incident sound waves and the animal's head and external ears. In barn owls, these cues include frequency-specific interaural time differences (ITDs), which vary primarily with sound source azimuth,

\footnotetext{
Received June 3, 1999; revised Oct. 8, 1999; accepted Oct. 29, 1999.

This work was supported by the National Institute on Deafness and Other Communication Disorders, National Institutes of Health Grant R01 DC00155-18. We thank Bob Schneeveis for helping to design and construct the acoustic filtering device and Greg Miller, Fred Rieke, Ed Rubel, and Michael Shadlen for helpful comments on this manuscript.

Correspondence should be addressed to Joshua I. Gold, Department of Physiology and Biophysics, University of Washington Medical School, Box 357290, Seattle, WA 98195-7290. E-mail: jig@u.washington.edu.

Dr. Gold's present address: Department of Physiology and Biophysics, University of Washington, Seattle, WA 98195-7290.

Copyright (C) 2000 Society for Neuroscience $\quad 0270-6474 / 00 / 200862-16 \$ 15.00 / 0$
}

reflecting the acoustic effects of the device. ILD tuning was shifted in the adaptive direction by $\sim 3 \mathrm{~dB}$ for $4 \mathrm{kHz}$ stimuli and $8 \mathrm{~dB}$ for $8 \mathrm{kHz}$ stimuli, but these shifts were substantially smaller than expected based on the acoustic effects of the device. Most sites also exhibited conspicuously abnormal frequency-response functions, including a strong dependence on stimulus ITD and a reduction of normally robust responses to 6 $\mathrm{kHz}$ stimuli. The results demonstrate that the response properties of high-order auditory neurons in the optic tectum are adjusted during development to reflect the influence of frequency-specific features of the binaural localization cues experienced by the individual.

Key words: sound localization; hearing impairment; development; auditory plasticity; superior colliculus; Tyto alba

and level differences (ILDs), which vary primarily with sound source azimuth for frequencies below $\sim 4 \mathrm{kHz}$ but with elevation for higher frequencies (attributable to an asymmetry in the owl's external ears). Previous work has demonstrated that adaptive modification of tectal unit auditory receptive fields (RFs) corresponds to changes in their tuning for broadband ITD and ILD. For example, either monaural occlusion with a dense foam plug or removal of the sound collecting structures of the external ears alters sound timing and level and leads to adaptive changes in the values of broadband ITD and ILD to which tectal units are tuned (Mogdans and Knudsen, 1992; Knudsen et al., 1994). Likewise, experience with a prismatically displaced visual field during development leads to a shift in tectal auditory RFs as units become tuned to the values of localization cues that are produced by sound stimuli located in their optically displaced visual RFs (Brainard and Knudsen, 1993).

We investigated the extent to which the binaural tuning properties of tectal neurons accurately reflect frequency-specific features of the individual's auditory experience. In a previous study, we caused frequency-dependent changes in the auditory localization cues experienced by young owls by raising them with an acoustic filtering device in one ear. We found frequency-specific changes in the auditory RFs of tectal units that compensated for the acoustic effects of the device (Gold and Knudsen, 1999). In the present study, we used the same sensory manipulation and characterized the resulting adaptive adjustments in terms of frequency-dependent changes in the tuning of tectal units for binaural localization cues.

\section{MATERIALS AND METHODS}

We collected data from eight normal barn owls (Tyto alba) and eight barn owls raised with an acoustic filtering device in one ear. Four of the 
device-reared owls and two of the normal owls were also used in a previous study (Gold and Knudsen, 1999).

Auditory experience. The acoustic filtering device used to alter auditory experience was a custom-designed chamber made from acetal delrin (Plastics SRT) that was sutured into the owl's right ear canal, just behind the preaural flap and in front of the sound-collecting surface formed by the facial ruff feathers (the owl's ear canals are asymmetrically positioned on its head, and the left ear canal opens at an angle relative to the facial ruff feathers that makes it difficult to place the device on the left side). We designed the device to increase the path length of sounds reaching the affected ear and to change the resonance properties of the ear canal while still providing a low impedance pathway to the tympanic membrane. The device is described in more detail in a previous study (Gold and Knudsen, 1999), which also includes cochlear microphonic measurements of its effects on the timing and level of sounds reaching the affected ear.

Owls raised with an acoustic filtering device were first binaurally occluded from 25-35 d of age with dense foam rubber plugs (E.A.R. Cabot Corporation) to disrupt early auditory experience while the ear canals were open but too narrow to hold the device. The plugs were sutured into the ear canals while the animal was anesthetized with halothane $(1 \%)$ in a mixture of oxygen and nitrous oxide (5:4). At $\sim 35$ $\mathrm{d}$ of age, the owl was again anesthetized, the binaural foam plugs were removed, and the filtering device was sutured into the right ear canal.

All owls were raised initially in brooding boxes with their siblings. After device insertion, owls were removed from the brooding box and were placed into a small cage located next to a large flight cage that housed adult owls, to ensure a rich auditory and visual environment. When the owls could fly (normally at $\sim 60 \mathrm{~d}$ of age), they were transferred to the flight cage, where they were housed for the duration of the experiment.

The acoustic device was removed only during experiments, at which time the canal, eardrum, and device were inspected for damage and cleaned of earwax. The device was sutured back into place immediately after each experiment.

The owls were provided for in accordance with the National Institutes of Health Guide for the Care and Use of Laboratory Animals and the guidelines of the Stanford University Institutional Animal Care and Use Committee.

Electrophysiology. Electrophysiological recordings were performed on owls that were at least 95-d-old. Each owl was prepared for recordings in a single surgical session, at which time the owl was anesthetized with halothane and nitrous oxide, and a small, stainless steel plate was cemented to the base of the skull. In addition, small craniotomies were made above the left and right optic tecta. These openings were covered with dental acrylic, which was removed and replaced for each recording session. At the end of the procedure, lidocaine was infused into any incised tissue, and all incisions were treated with betadine and sutured closed.

At the beginning of each recording session, the owl was anesthetized with halothane and nitrous oxide, given an intramuscular injection of $2.5 \%$ dextrose in $0.45 \%$ sterile saline, and wrapped in a soft leather jacket. The owl was then suspended in a prone position in a stereotaxic apparatus that was centered in a sound attenuating chamber (Industrial Acoustics Company 404A) lined with acoustic foam to suppress echoes. The owl's head was bolted to the stereotaxic apparatus via the surgically implanted steel plate and aligned using retinal landmarks (a barn owl's eyes are essentially fixed in its head). A tungsten microelectrode was positioned and then advanced through the telencephalon to the tectum using a mechanical microdrive. A characteristic bursting pattern of unit activity indicated when the electrode tip entered the superficial layers of the optic tectum (Knudsen, 1982), where all recordings were made.

Other than the initial $20 \mathrm{~min}$ of set-up, the owl typically remained unanesthetized and was calm and motionless during the course of the experiment. Occasionally, however, an owl became restless, in which case recording was suspended temporarily and brief doses of halothane and nitrous oxide or nitrous oxide alone were administered.

After each experiment, the owl recovered under a heat lamp overnight before being returned to the flight cage.

Auditory measurements. Dichotic stimuli consisted of computergenerated broadband, narrowband, or tonal bursts lasting $50 \mathrm{msec}$. The stimuli were presented through matched Knowles subminiature earphones (ED-1914) coupled to damping assemblies (BF-1743) placed in each ear $\sim 5 \mathrm{~mm}$ from the tympanic membrane. The amplitude and phase spectra of the earphones were equalized to within $\pm 2 \mathrm{~dB}$ and $\pm 2 \mu \mathrm{sec}$ from 1 to $12 \mathrm{kHz}$ by computer adjustments. Sound levels were calibrated using A-weighted signals from a Bruel and Kjær $1 / 2$ inch condenser microphone positioned $1 \mathrm{~cm}$ from the earphone. Broadband noise bursts had rise-fall times of $0 \mathrm{msec}$ and had a passband of $3-12 \mathrm{kHz}$, the lower bound set to minimize propagation through the interaural canal (Moiseff and Konishi, 1981). Unless otherwise indicated, narrowband stimuli were generated with a 1-kHz-wide digital filter centered on the given frequency. Narrowband and pure tone bursts had rise-fall times of $5 \mathrm{msec}$. In figures, the passband of a given stimulus is indicated as "low-high", whereas the range of center stimulus frequencies used in a given analysis of pooled data are indicated as "(low, high)."

A level discriminator was used to isolate action potentials ("spikes") generated by a small number of tectal neurons. Spike times relative to the onset of a given sound presentation were stored on a computer. The response to a sound presentation was defined as the number of spikes counted in the $100 \mathrm{msec}$ immediately after stimulus onset (poststimulus response) minus the number of spikes counted in the $100 \mathrm{msec}$ immediately preceding stimulus onset (baseline activity).

At each site, we characterized response thresholds, frequency responses, and ITD and ILD tuning using broadband and narrowband stimuli. Response threshold was defined as the lowest average binaural level at which responses to a given stimulus were at least $25 \%$ of the maximum responses produced by that stimulus for any average level up to $70 \mathrm{~dB}$ SPL. Frequency, ITD, and ILD tuning curves were characterized by calculating the widths and best values of tuning curve peaks. For each tuning curve, the maximum response was determined, and the width of the peak was defined as the uninterrupted range over which responses were $>50 \%$ of the maximum. The midpoint of that range was defined as the best value for that peak. For some tuning curves, responses to values outside of the peak containing the maximum response exceeded $50 \%$ of the maximum response. The widths and best values from all such peaks were determined separately.

Frequency-response functions were measured using tonal and $1 \mathrm{kHz}$ bandwidth stimuli. Tone bursts were presented using the best ITD and best ILD measured for the broadband stimulus and at $\sim 20 \mathrm{~dB}$ above the threshold measured for the lowest frequency above $3 \mathrm{kHz}$ that elicited a response. Stimuli of $1 \mathrm{kHz}$ bandwidth were presented using the best ITD and best ILD and at 20-30 $\mathrm{dB}$ above the threshold measured for the given stimulus.

ITD tuning was measured at 10-20 $\mu \mathrm{sec}$ intervals over a 100-300 $\mu \mathrm{sec}$ range of values (positive and negative values indicate right- and left-ear leading, respectively). Each ITD value was presented at the estimated best ILD and at 20-30 $\mathrm{dB}$ above the threshold measured for the given stimulus.

ILD tuning was measured at 2-4 dB intervals over a 15-40 dB range of values (positive and negative values indicate right- and left-ear greater, respectively). Each ILD value was presented at the best ITD and at a constant average binaural level 20-30 dB above the threshold measured for the given stimulus. For sites with ITD tuning curves with multiple peaks for a broadband stimulus, ILD tuning was measured using the best ITD for each peak.

Visual receptive field measurements. Visual RFs were measured by projecting bars and spots of light onto a translucent hemisphere placed in front of the owl. Visual RF location is reported as the geometric center of the RF in a double-pole coordinate system (Knudsen, 1982), in which azimuth indicates degrees right $(\mathrm{R})$ or left $(\mathrm{L})$ of the midsagittal plane and elevation indicates degrees above $(+)$ or below $(-)$ the visual (horizontal) plane.

Comparison with expected values of ITD and ILD. Best ITDs and best ILDs were compared to the values predicted for sites with matching visual RF locations in normal owls. For broadband stimuli, predicted normal best ITD and best ILD values were determined from previously published linear regressions that describe the predictable relationships between best values and visual RF location in the optic tecta of normal owls (ITD, Brainard and Knudsen, 1993; ILD, Mogdans and Knudsen, 1992). For narrowband stimuli, predicted normal best ITD and best ILD values were the acoustic values of ITD and ILD, respectively, produced by that center frequency when the source was located in the visual RF of the site. The values used were the means of probe tube microphone measurements from five different owls (Knudsen et al., 1991; S. Esterly, personal communication).

\section{RESULTS}

We examined the extent to which early experience with the chronically implanted acoustic device altered the binaural tuning properties of tectal neurons by comparing the frequency re- 


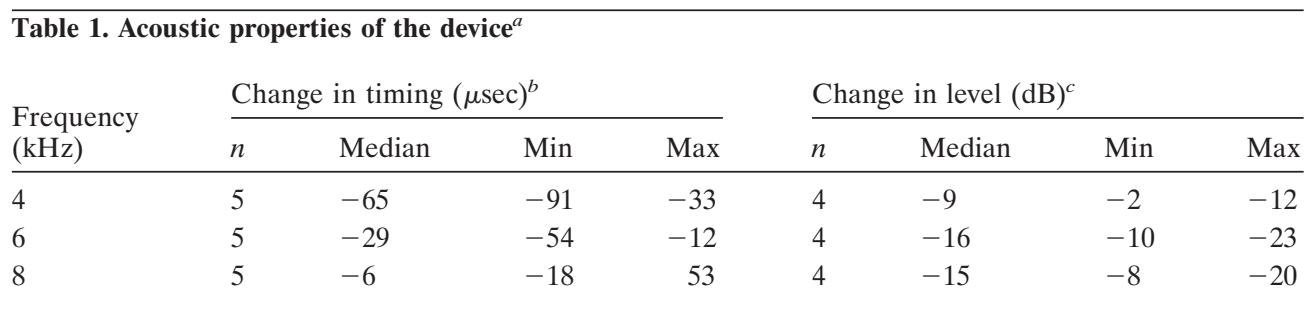

${ }^{a}$ Cochlear microphonic measurements of the difference in the timing and level of sounds reaching the eardrum before versus after device insertion, with the sound source located at $0^{\circ}$ az, $0^{\circ}$ el (Gold and Knudsen, 1999).

${ }^{b}$ Time difference nearest $0 \mu \mathrm{sec}$ for the measured phase difference; negative values indicate delays.

${ }^{c}$ Negative values indicate attenuations.
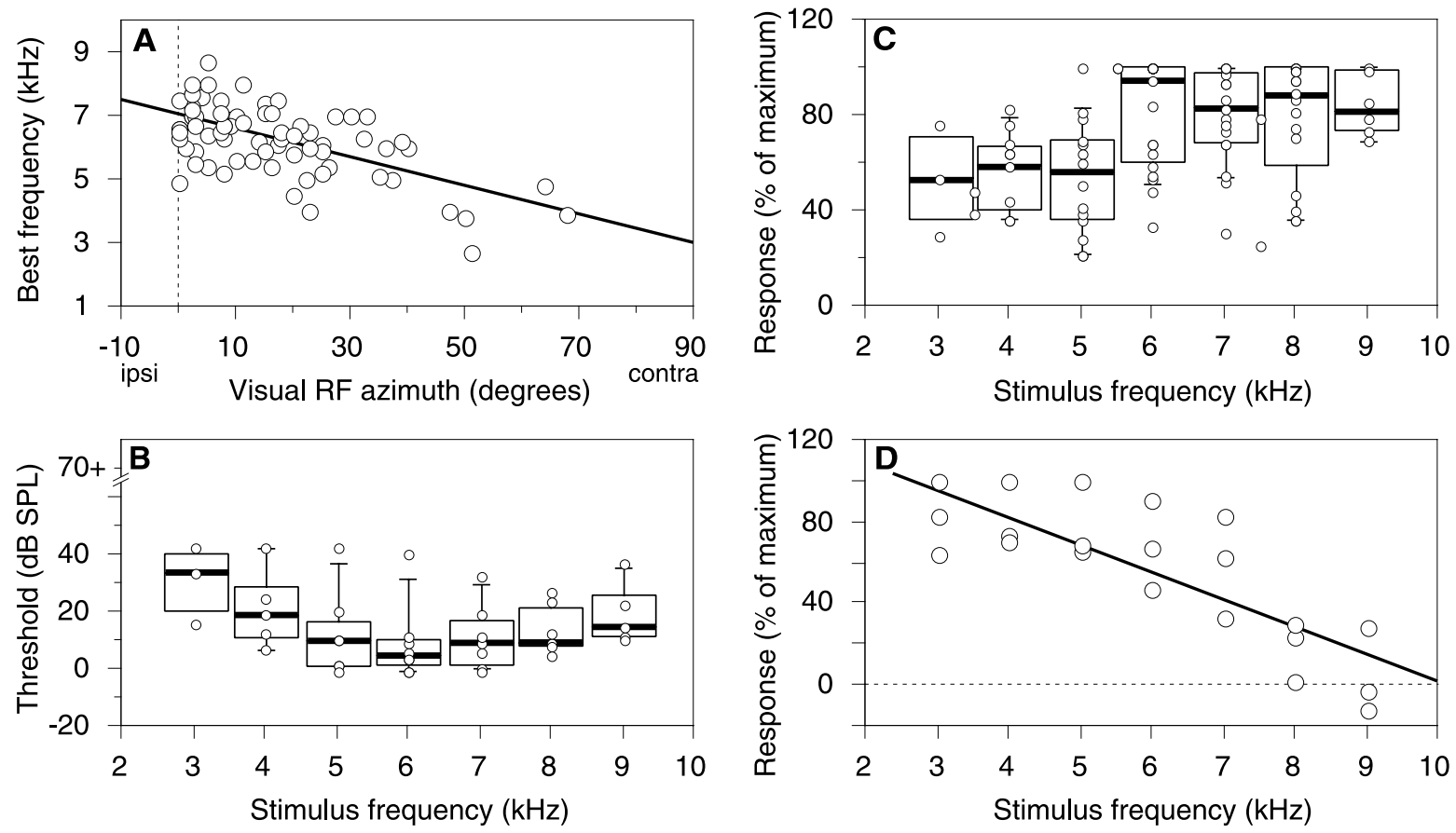

Figure 1. Frequency tuning in normal owls. $A$, Best frequency as a function of visual RF azimuth. Responses were measured using tonal stimuli and the broadband best ITD-ILD pair for the given site. The solid line is a linear fit to the data $\left(y=-0.04 \mathrm{x}+7.0 ; r^{2}=0.38\right.$; ANOVA, $\left.p<0.001\right)$. B, Response threshold as a function of the center frequency of the narrowband (1 kHz bandwidth) stimulus. Responses were measured using the best ITD-ILD pair for the given stimulus at sites with visual RFs between $\mathrm{L} 25^{\circ}$ and R $25^{\circ}$ az. " $70+$ " indicates that no reliable responses could be elicited using the given stimulus presented at sound levels up to $70 \mathrm{~dB}$ SPL. Thick lines, boxes, and bars indicate medians, quartiles, and $10^{\text {th }}-90^{\text {th }}$ percentiles, respectively, of binned data. $C$, Strength of response as a function of the center frequency of the narrowband (1 kHz bandwidth) stimulus, normalized to the maximum response elicited by any $1-\mathrm{kHz}$-wide stimulus at the same recording site. Responses were measured as in $B$ at sites with visual RFs between $\mathrm{L} 25^{\circ}$ and $\mathrm{R} 25^{\circ}$ az. Thick lines, boxes, and bars as in $B . D$, Data measured as in $C$, but at sites with visual RFs more peripheral than $25^{\circ}$ az. The solid line is a linear fit to the data $\left(y=-13.5 \mathrm{x}+136.9 ; r^{2}=0.65\right.$; ANOVA, $\left.p<0.001\right)$.

sponses and the narrowband and broadband ITD and ILD tuning of tectal neurons in normal and device-reared owls. The effects of the device on the timing and level of sound reaching the right eardrum were measured in a previous study using cochlear microphonics (Gold and Knudsen, 1999). Table 1 summarizes these effects for sound sources located directly in front of the owl. In addition, by interfering with the directional sensitivity of the external ears, the device affected sound in a direction-dependent manner, which was particularly evident in a reduced attenuation at all frequencies for sources located to the right.

\section{Frequency-response functions in normal owls}

Frequency-response functions, when measured using dichotic stimuli with constant ITD and ILD values, reflect both the spectral sensitivity of a unit and its tuning for binaural cues. The effect of binaural tuning on dichotically measured frequency-response functions is eliminated when the binaural tuning of a unit is independent of frequency. Therefore, to minimize the effect of binaural tuning on frequency-response functions measured using constant ITD and ILD values in normal owls, we sampled frequency responses primarily from the rostral tectum, which represents frontal space where sounds produce nearly constant values of ITD and ILD across frequency (Knudsen et al., 1991). Some sites representing peripheral locations were also sampled.

Figure $1 A$ depicts frequency-response functions of tectal neurons measured dichotically, using tonal stimuli with the best ITD and best ILD values measured with a broadband stimulus for the given site. These frequency-response functions were consistently broad (width $=3.1 \pm 1.1 \mathrm{kHz}$, mean $\pm \mathrm{SD}$ ), a characteristic known to play a key role in resolving the spatial ambiguities that are inherent to sound localization cues (Brainard et al., 1992). 
Best frequencies tended to decrease as a function of visual RF azimuth, from an average of $6.5 \mathrm{kHz}$ for units representing frontal space to $<5 \mathrm{kHz}$ for units representing peripheral space. The same relationship was reported previously in studies that used free field stimulation, for which the owl's head and ears shaped ITDs and ILDs naturally (Knudsen, 1984; Olsen et al., 1989). This progression of best frequencies with sound source azimuth parallels a decrease in the sensitivity of the ears to high-frequency stimuli as the source moves peripherally (Payne, 1971; Knudsen, 1980).

To account for possible effects of stimulus ITD and ILD on frequency-response functions, we also assessed frequency responses using the best ITD and best ILD measured for each stimulus. Because responses to tones were often weak, we measured these frequency responses using stimuli with a bandwidth of $1 \mathrm{kHz}$, which typically elicited much stronger responses. For frontal sites, with visual RF azimuths between $\mathrm{L} 25^{\circ}$ and $\mathrm{R} 25^{\circ}$, responses to these stimuli were typically strong for all frequencies between 3 and $9 \mathrm{kHz}$. Responses were strongest and thresholds were lowest, however, for stimuli near $6 \mathrm{kHz}$ (Fig. 1B,C). In contrast, for sites with more peripherally located visual RFs, the strongest responses were elicited by lower frequencies (Fig. 1D).

\section{Frequency-response functions in device-reared owls}

Because the acoustic device altered ITD and ILD differently for different frequencies, an adaptive adjustment to the effects of the device would require the ITD and ILD tuning even of frontal units to vary commensurately with stimulus frequency. This type of adjustment would cause the frequency tuning of these units, measured with dichotic stimuli, to vary dramatically depending on the values of ITD and ILD that were chosen for the stimulus. Indeed, such ITD- and ILD-dependent frequency-response functions were observed routinely among frontal units in devicereared owls (Fig. 2).

In the example shown in Figure $2 A$, the frequency-response curve for a tectal site with a visual RF at $\mathrm{L}^{\circ}$ azimuth $(\mathrm{az}),+10^{\circ}$ elevation (el) in a device-reared owl had a best value of $4.0 \mathrm{kHz}$ when measured with an ITD of $-60 \mu \mathrm{sec}$ but a best value of 7.5 $\mathrm{kHz}$ when measured with an ITD of $+30 \mu$ sec. In general, frequency tuning curves had best values of $\sim 4 \mathrm{kHz}$ when measured using a stimulus ITD that was offset from the predicted normal best ITD by between 20 and $100 \mu$ sec toward left-ear leading. These shifted ITDs roughly match the acoustic delay imposed by the device at $4 \mathrm{kHz}$. In contrast, frequency tuning curves typically had best values clustered around $7.5 \mathrm{kHz}$ when measured using a stimulus ITD that was offset from the predicted normal best ITD either slightly toward right-ear leading or by $\sim 100 \mu \mathrm{sec}$ toward left-ear leading. The period of $7.5 \mathrm{kHz}$ is 133 $\mu \mathrm{sec}$, so the peaks of this bimodal distribution represent a similar value of interaural phase difference at $7.5 \mathrm{kHz}$. This interaural phase difference roughly matches the small phase shift imposed by the device near $7.5 \mathrm{kHz}$.

The frequency-response functions measured in device-reared owls using the broadband best ITD and best ILD values for the given site were abnormal (Fig. $2 C$ ). As in normal owls, best frequency varied with visual RF azimuth such that, on average, best frequencies were higher for sites with frontally located visual RFs. In contrast to the normal distribution of best frequencies found for sites representing frontal space in normal owls (Fig. $1 A$ ), however, the distribution of best frequencies for comparable sites in device-reared owls was bimodal, with most best frequencies clustered near 4 and $7.5 \mathrm{kHz}$ and few between 5 and $6 \mathrm{kHz}$.
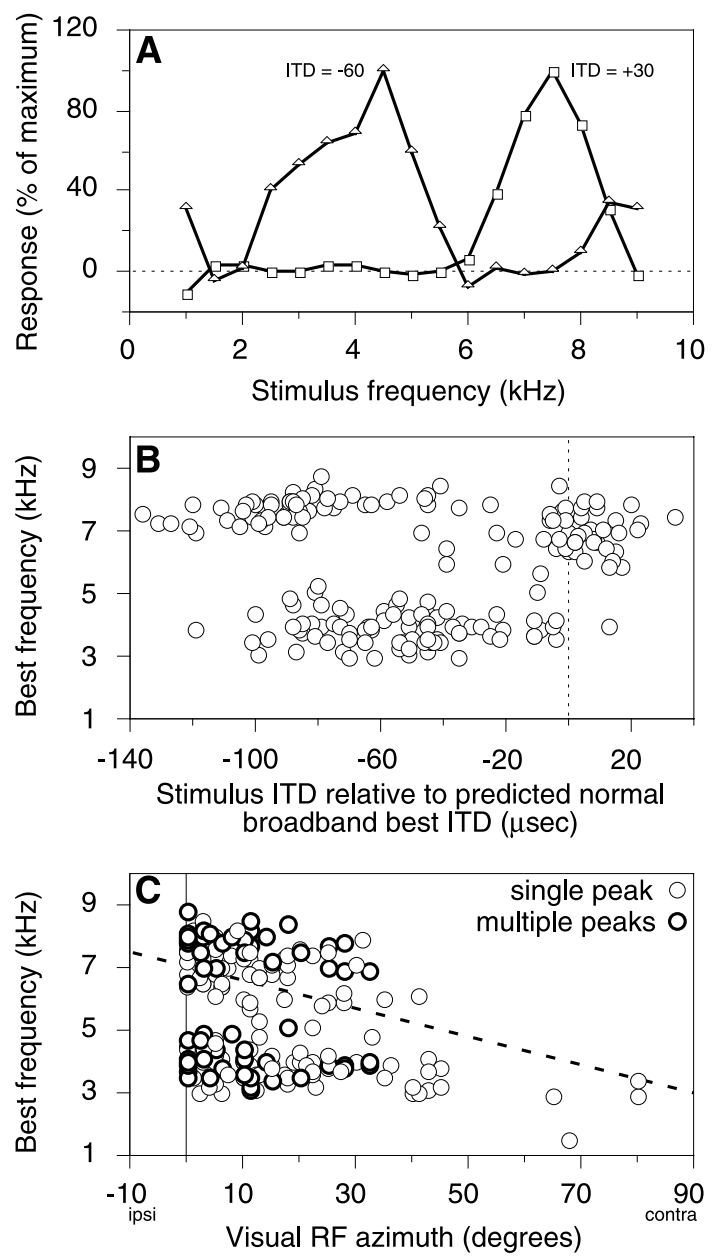

Figure 2. Frequency tuning in device-reared owls. Responses were measured using tonal stimuli. $A$, Two frequency-response curves from a tectal site with a visual $\mathrm{RF}$ at $\mathrm{L} 2^{\circ}$ az, $+10^{\circ}$ el in a device-reared owl. The two curves were measured under identical conditions except for the stimulus ITD used, as indicated. $B$, Best frequency as a function of the stimulus ITD relative to the predicted normal broadband best ITD. The predicted normal broadband best ITD was determined from the visual RF azimuth (see Materials and Methods). The stimulus ITD used was the broadband best ITD at the given site; for sites with multiple best ITDs, frequency tuning was measured using each best ITD separately. Best values from all peaks of all curves measured at sites with visual RFs between $\mathrm{L} 25^{\circ}$ and $\mathrm{R} 25^{\circ}$ az are shown. $C$, Best frequency as a function of visual RF azimuth. The dashed line is a linear fit to the data from normal owls (Fig. 1A). For frequency tuning curves with more than one peak, best values from both peaks are shown in bold.

Indeed, many of the frequency-response curves measured at sites with frontally located visual RFs were themselves bimodal, with strong responses near 4 and $8 \mathrm{kHz}$ and weak responses near $6 \mathrm{kHz}$.

The dependence of best frequency on stimulus ITD in devicereared owls implies frequency-dependent changes in ITD tuning. Thus, the abnormal frequency-response functions using constant ITD and ILD values likely reflected the effects of nonoptimal binaural cue values at particular frequencies. However, as shown in Figures 3 and 4, frequency-response functions measured using the best ITD and best ILD for each narrowband (1-kHz-wide) stimulus revealed that the device did, in addition, induce abnormal spectral sensitivity. In particular, tectal units representing frontal space in device-reared owls exhibited a substantial reduc- 

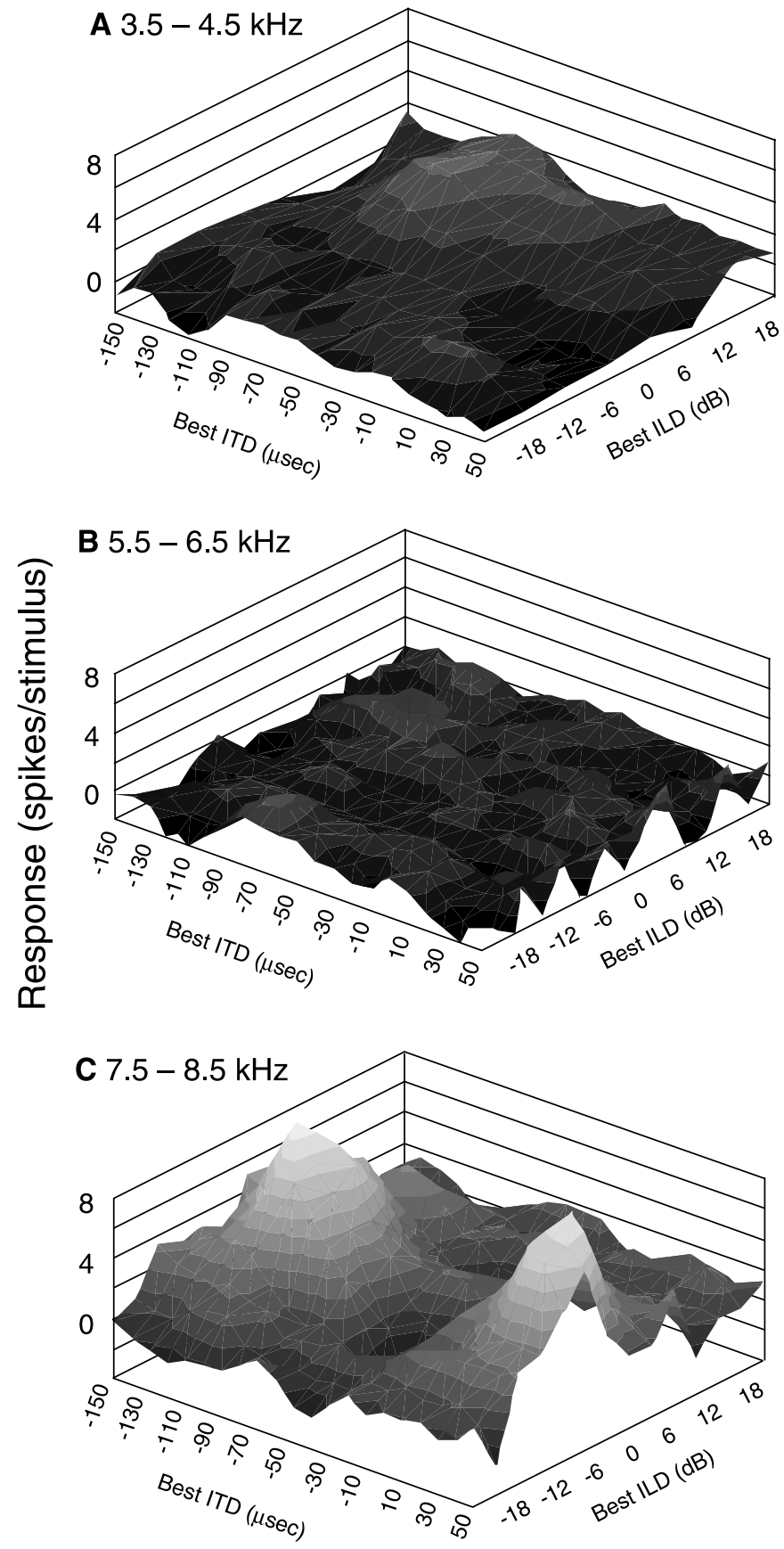

Figure 3. Responses to narrowband (1-kHz-wide) stimuli centered on 4 $(A), 6(B)$, or $8(C) \mathrm{kHz}$ for a tectal site in a device-reared owl with a visual $\mathrm{RF}$ at $0^{\circ} \mathrm{az},+3^{\circ}$ el. $A$, Maximum response $=3.2$ spikes per stimulus at ITD $=-72 \mu \mathrm{sec}, \mathrm{ILD}=+3 \mathrm{~dB} . B$, Maximum response $=0.8$ spikes per stimulus at ITD $=+50 \mu \mathrm{sec}, \mathrm{ILD}=+6 \mathrm{~dB}$ (presented at $30 \mathrm{~dB}$ above the threshold measured with the $4 \mathrm{kHz}$ stimulus). $C$, Maximum response $=6.2$ spikes per stimulus at ITD $=-106 \mu \mathrm{sec}$, ILD $=+3 \mathrm{~dB}$.

tion in responses to stimuli near $6 \mathrm{kHz}$. These changes match those measured using free field stimulation, as reported in a previous study (Gold and Knudsen, 1999).

Figure 3 illustrates responses for a single site (visual RF at $0^{\circ}$ $\left.\mathrm{az},+3^{\circ} \mathrm{el}\right)$ in a device-reared owl for narrowband stimuli centered on 4, 6 , and $8 \mathrm{kHz}$. For this site, the best ITD-ILD pair for the $8 \mathrm{kHz}$ stimulus elicited the strongest responses, which were nearly twice as strong as those elicited by the best ITD-ILD pair
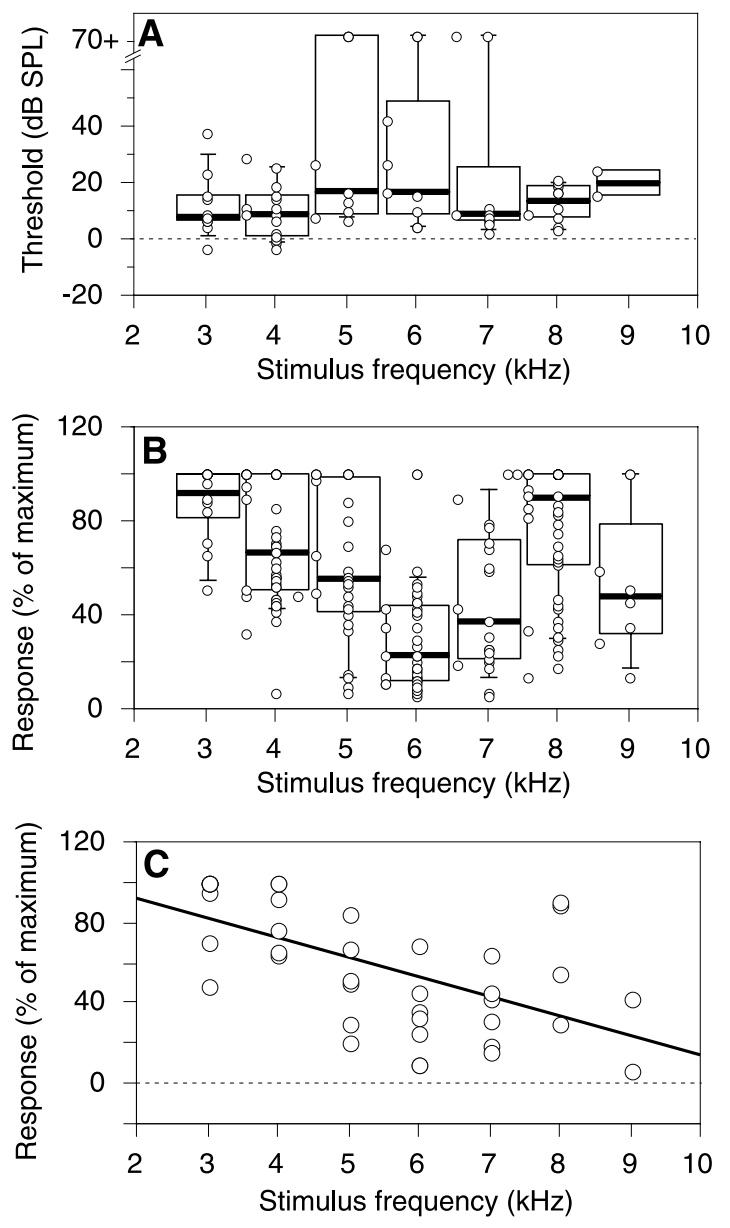

Figure 4. Summary of responses to narrowband (1-kHz-wide) stimuli in device-reared owls. All responses were measured using the best ITDILD pair for the given stimulus. $A$, Response threshold as a function of the center frequency of the stimulus for sites with visual RFs between $\mathrm{L} 25^{\circ}$ and $\mathrm{R} 25^{\circ}$ az. "70+" indicates that no reliable responses could be elicited using the given stimulus presented at sound levels up to $70 \mathrm{~dB}$ SPL. Thick lines, boxes, and bars indicate medians, quartiles, and $10^{\text {th }}$ $90^{\text {th }}$ percentiles, respectively, of binned data. $B$, Strength of response as a function of the center frequency, normalized to the maximum response elicited by any such narrowband stimulus at the same recording site, for sites with visual RFs between $\mathrm{L} 25^{\circ}$ and $\mathrm{R} 25^{\circ}$ az. Thick lines, boxes, and bars as in $A$. $C$, Data measured as in $B$, but at sites with visual RFs more peripheral than $25^{\circ}$ az. The solid line is a linear fit to the data $(y=-10.0 \mathrm{x}$ $+111.5 ; r^{2}=0.34$; ANOVA, $\left.p<0.001\right)$.

for the $4 \mathrm{kHz}$ stimulus. In contrast, no ITD-ILD pair for the 6 $\mathrm{kHz}$ stimulus elicited robust responses. Similar results were found for sites with visual RF azimuths between $\mathrm{L} 25^{\circ}$ and $\mathrm{R} 25^{\circ}$. For these sites, response thresholds were relatively normal for stimuli near 4 and $8 \mathrm{kHz}$ but not for stimuli near $6 \mathrm{kHz}$, which in some cases did not elicit any responses for sound levels up to $70 \mathrm{~dB}$ SPL (Fig. $4 A$ ). Responses to 4 and $8 \mathrm{kHz}$ stimuli were strong, but responses to stimuli near 6 and $7 \mathrm{kHz}$ were typically $<40 \%$ of the maximum response elicited by any narrowband stimulus at a given site, which was significantly weaker than normal (MannWhitney $U$ test, $p<0.01$; Fig. $4 B$ ). For sites with more peripherally located visual RFs, frequency responses were relatively normal, however, with the strongest responses to stimuli near 3 and $4 \mathrm{kHz}$ and weaker responses to higher frequencies (Fig. 4C). 


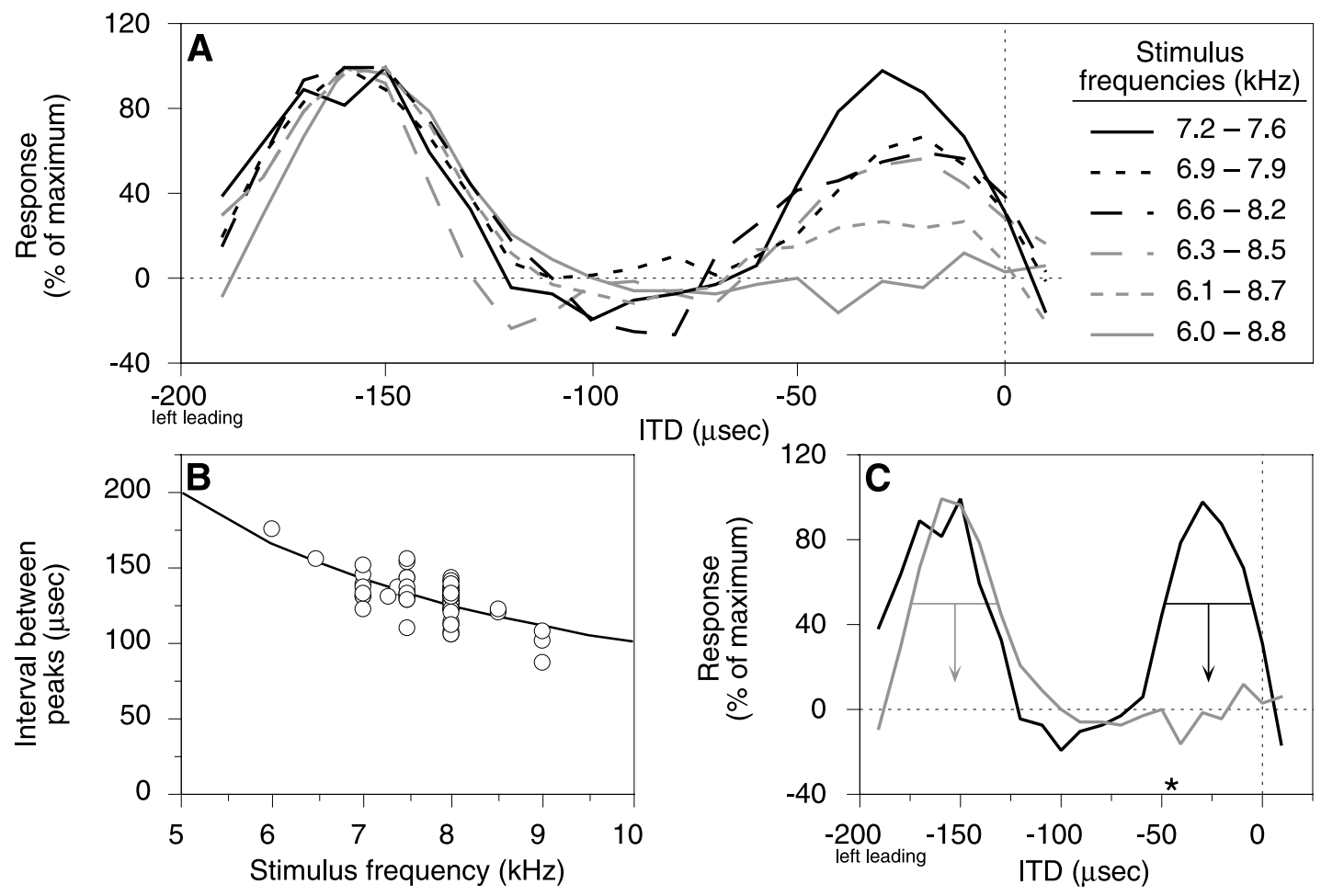

Figure 5. Effect of stimulus bandwidth on ITD tuning curves in the optic tectum of a device-reared owl. $A$, ITD tuning curves measured using stimuli centered on $7.4 \mathrm{kHz}$ (the best frequency of the site) with a variety of bandwidths (see key for the ranges of stimulus frequencies used). $B$, The interval between adjacent peaks for all narrowband ITD tuning curves from both normal and device-reared owls that had multiple peaks. The data are plotted as a function of the center frequency of the stimulus. The solid line indicates the interval (in microseconds) that represents an interaural phase offset of $360^{\circ}$. $C$, ITD tuning curves from $A$ measured using either a $7.2-7.6 \mathrm{kHz}$ (solid line) or a $6.0-8.8 \mathrm{kHz}$ (shaded line) narrowband stimulus. Best ITD (downward arrow in each curve) was computed as the midpoint of the response peak closest to the predicted normal value (asterisk), which was based on the visual RF location.

\section{Bandwidth for testing narrowband tuning}

We used primarily stimuli with a bandwidth of $1 \mathrm{kHz}$ to characterize the frequency-dependent effects of device rearing on the ITD and ILD tuning of tectal neurons. Stimuli with this bandwidth elicited stronger responses than did pure tones and provided adequate specificity to characterize differences in binaural tuning for different stimulus frequencies (Fig. 3). However, nontonal stimuli can trigger inhibitory interactions across frequency channels (Takahashi and Konishi, 1986) that affect tuning curve shape. For example, ITD tuning curves are periodic when measured using tonal stimuli, because of the ambiguity of interaural phase with respect to ITD. In contrast, as illustrated in Figure 5 stimuli with more bandwidth yielded ITD tuning curves with a single, prominent peak, and the side peaks suppressed by crossfrequency inhibition. Even stimuli with a bandwidth of $1 \mathrm{kHz}$ in some cases yielded single-peaked ITD tuning curves (see Fig. 11C). In most cases, however, 1-kHz-wide stimuli yielded ITD tuning curves that, like those measured with tones, contained multiple peaks that were separated by ITD intervals equal to the period of the center frequency of the stimulus (Fig. $5 B$ ). For all ITD tuning curves, even those with multiple peaks, the best ITD was determined from the peak closest to the predicted normal value, which was based on the location of the visual RF of the site (Fig. 5C; see Materials and Methods).

\section{ITD tuning in normal owls}

We measured ITD tuning at tectal sites with visual RFs between $\mathrm{L} 30^{\circ}$ and $\mathrm{R} 41^{\circ}$ azimuth and $-15^{\circ}$ and $+12^{\circ}$ elevation in eight normal owls. These sites represent regions of space for which ITD varies systematically with source azimuth (Olsen et al., 1989) but does not vary substantially with frequency (Knudsen et al., 1991), properties that were reflected in their broadband and narrowband ITD tuning.

Figure 6 shows ITD tuning for broadband and narrowband stimuli for a tectal site with a visual $\mathrm{RF}$ at $\mathrm{L} 2^{\circ} \mathrm{az},+10^{\circ} \mathrm{el}$. For this site, broadband ITD tuning was narrow and had a best value of $-7 \mu$ sec. Similarly, each narrowband ITD tuning curve had a peak located near $-7 \mu \mathrm{sec}$, matching not only the broadband best ITD but the corresponding acoustic ITDs produced by a source located in the visual RF of the site, as well (Fig. 6B).

In agreement with previously published reports (Olsen et al., 1989; Brainard and Knudsen, 1993), tectal units from all sites tested in normal owls were sharply tuned for ITD using broadband stimuli (the $50 \%$ width had a mean value of $38 \mu \mathrm{sec}$ for 28 sites). Moreover, best ITD varied systematically and predictably with visual RF azimuth: for 46 sites tested, broadband best ITDs were within $20 \mu \mathrm{sec}$ of a linear fit to previously published best values plotted as a function of visual RF azimuth (best ITD $=2.5$ * visual RF azimuth; Brainard and Knudsen, 1993). Thus, in normal owls, visual RF azimuth accurately predicted unit ITD tuning for broadband stimuli.

For individual sites, the narrowband ITD tuning curves each had a peak that was aligned with the peak in the broadband ITD tuning curve. For 30 of 32 sites, the broadband best ITD matched to within $10 \mu \mathrm{sec}$ at least one narrowband best ITD. Moreover, 

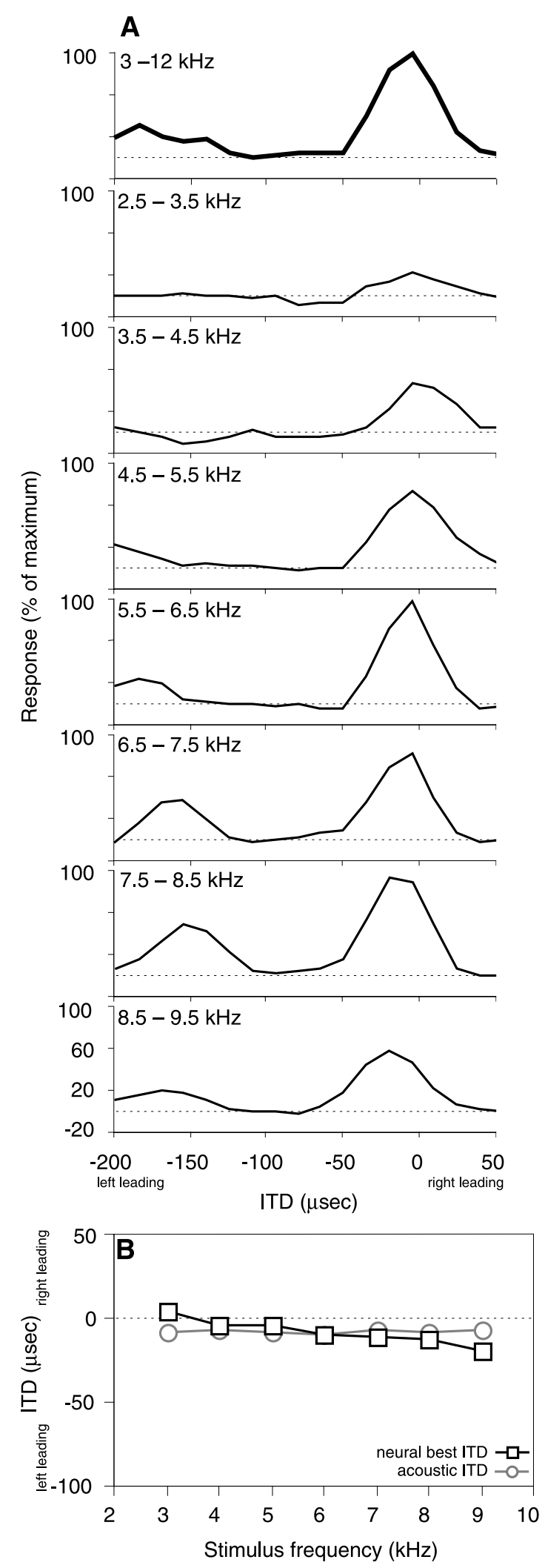

Figure 6. ITD tuning at a tectal site in a normal owl. The visual RF at this site was centered at $\mathrm{L} 2^{\circ}$ az, $+10^{\circ}$ el. $A$, ITD tuning curves obtained using broadband (3-12 kHz, top curve) or narrowband (the range of stimulus frequencies used is shown for each curve) stimuli. In some cases a second, smaller peak appeared when using a narrowband stimulus (e.g., stimulus $=6.5-7.5 \mathrm{kHz}$ ). In each case, the location of the second peak matched the expected location of an interaural phase equivalent peak for
102 of the 113 narrowband ITD tuning curves that we measured had a peak with a best value that was within the $50 \%$ cutoff limits of the broadband ITD peak.

Narrowband best ITDs in normal owls matched the acoustic ITDs produced by sources located at the visual RF of the site. For all 32 sites, narrowband best ITDs were relatively constant across the range of frequencies tested (Figs. 6, 7A). Across sites, narrowband best ITDs varied systematically with visual RF azimuth. As shown in Figure $7 B$, for example, best ITDs for narrowband stimuli centered on 4 and $8 \mathrm{kHz}$ progressed from left-ear leading to right-ear leading as visual RF azimuth moved from left to right. Linear fits to these data had slopes of slightly $>2 \mu \mathrm{sec} /{ }^{\circ}$ and intercepts of close to $0 \mu \mathrm{sec}$ at $0^{\circ}$ azimuth. For all sites and stimulus frequencies tested, the measured best ITD matched to within $30 \mu \mathrm{sec}$ the value predicted from the visual RF azimuth (Fig. 7C).

\section{ITD tuning in device-reared owls}

We measured ITD tuning at sites with visual RFs between $\mathrm{L} 81^{\circ}$ and $\mathrm{R} 60^{\circ}$ az and $-20^{\circ}$ and $+36^{\circ}$ el in eight device-reared owls. These sites represented regions of space for which, with the device in place, ITD varied with source azimuth and, unlike in normal owls, with frequency. Both broadband and narrowband ITD tuning at these sites were abnormal, mirroring frequencyspecific effects of the device on sound timing.

Figure 8 shows ITD tuning for broadband and narrowband stimuli for a site with a visual RF at $0^{\circ}$ az, $+8^{\circ}$ el in a devicereared owl. The broadband ITD tuning curve was broad and had a best value of $-41 \mu \mathrm{sec}$ (Fig. $8 A$, top curve). Narrowband ITD tuning depended strongly on the center frequency of the stimulus, unlike the tuning measured at sites with similar visual RFs in normal owls (Fig. 6). For example, relative to predicted normal values, best ITDs for stimuli near $4 \mathrm{kHz}$ were shifted by $\sim 50 \mu \mathrm{sec}$ toward left-ear leading, and best ITDs for stimuli around $8 \mathrm{kHz}$ were unshifted (Fig. 8B, squares). Although the narrowband best ITD values for many frequencies differed substantially from the acoustic ITDs experienced by normal owls (circles), they were close to the acoustic ITDs experienced by owls with the device in place (triangles). The tuning curves measured at this site differed further from normal in that the strongest responses were elicited by stimuli near 4 and $8 \mathrm{kHz}$, whereas stimuli near $6 \mathrm{kHz}$, which normally elicit strong responses, elicited almost no responses at any ITD (Figs. 1-4).

The effects of device rearing on broadband ITD tuning are summarized in Figure 9. Figure $9 A$ shows broadband best ITD as a function of visual RF azimuth for all sites tested in devicereared owls, whereas Figure $9 B-J$ highlights the device-induced changes in ITD tuning that were evident in different regions of the tectal space map. For sites representing space to the far left (visual $\mathrm{RF}$ az, $\geq \mathrm{L} 25^{\circ}$ ), broadband ITD tuning curves had similar shapes (Fig. 9B) as those found at corresponding sites in normal owls, but had best ITDs that were shifted from normal by $79 \pm 19$

$\leftarrow$

the center frequency of the given range (e.g., offset by integer multiples of $143 \mu \mathrm{sec}$ for a $7 \mathrm{kHz}$ tone). Broadband responses were normalized relative to the maximum response elicited with the broadband stimulus. Tuning curves for all narrowband stimuli were normalized relative to the maximum response elicited with any narrowband stimulus. $B$, Best values from the tuning curves in $A$ for narrowband stimuli plotted as a function of the center frequency (squares), along with the frequency-specific, average acoustic ITDs experienced by normal owls for sound sources located in the center of this visual RF of the site (circles). 

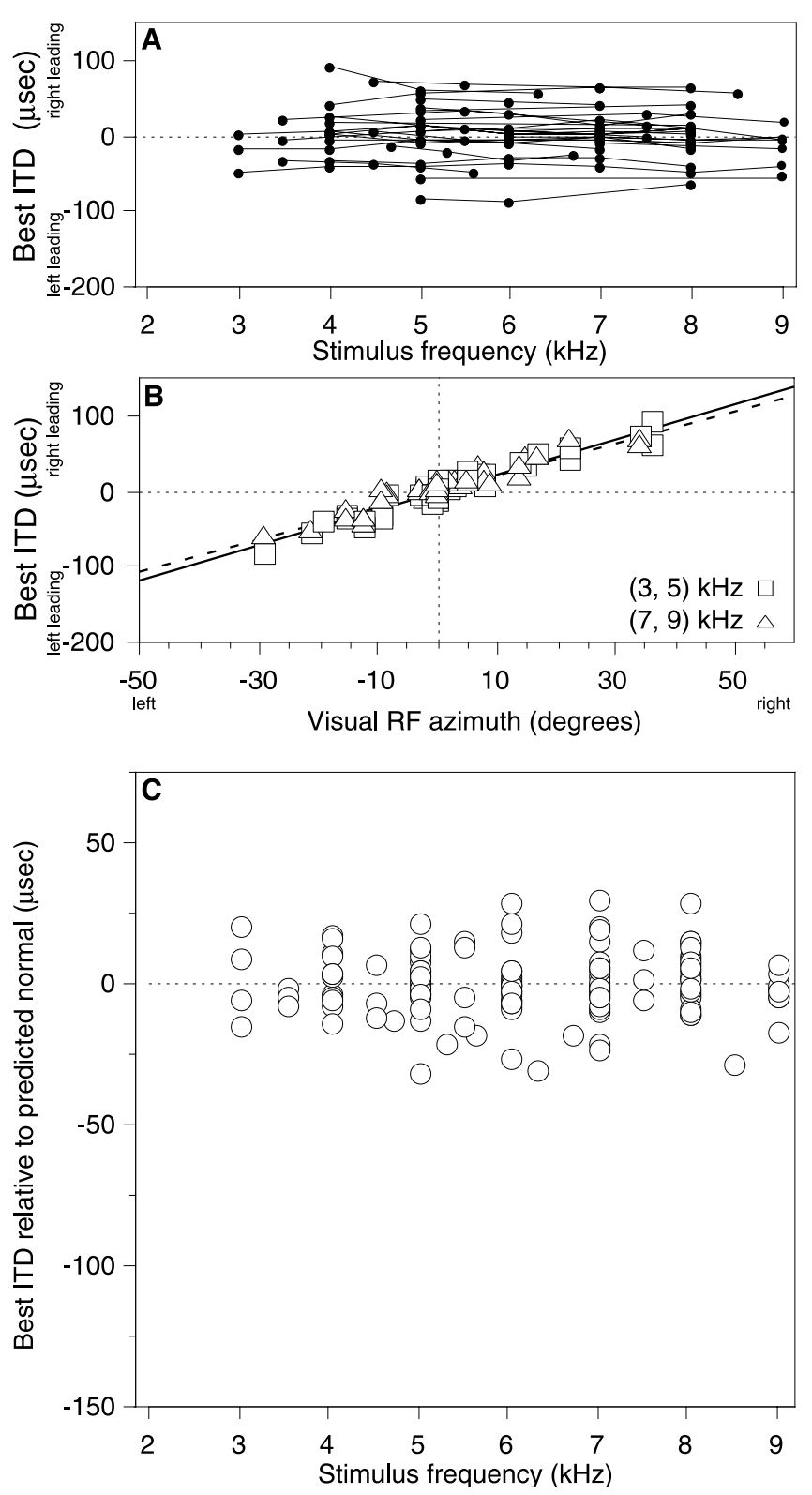

Figure 7. Summary of narrowband ITD tuning of tectal neurons in normal owls. Data are from 32 sites with visual RFs between $\mathrm{L} 30^{\circ}$ and $\mathrm{R} 37^{\circ}$ az and $-10^{\circ}$ and $+15^{\circ}$ el. $A$, Best ITD as a function of center stimulus frequency. Each line connects points representing measurements taken at a single recording site $(n=32)$. $B$, Best ITDs for low-frequency $(3,5 \mathrm{kHz})$, and high-frequency $(7,9 \mathrm{kHz})$ stimuli as functions of visual $\mathrm{RF}$ azimuth. Linear least-squares fits are shown for the low- (solid line; $y=$ $\left.2.3 \mathrm{x}-1.7 ; r^{2}=0.93\right)$ and high- (dashed line; $\left.y=2.1 \mathrm{x}-0.9 ; r^{2}=0.89\right)$ frequency data, both of which were highly significant (ANOVA, $p<$ $0.001)$. $C$, Best ITD relative to the acoustic ITD produced by a source located at the visual RF of the site as a function of the center stimulus frequency; the two were not correlated (ANOVA, $p=0.85$ ).

$\mu$ sec toward left-ear leading (mean \pm SD; Fig. 9E) and were broader than normal (Fig. $9 H$ ). For sites representing frontal space (visual RF az between $\mathrm{L}^{\circ} 5^{\circ}$ and $\mathrm{R} 25^{\circ}$ ), broadband tuning curves were often irregular in shape and typically contained multiple peaks (Fig. 9C, solid line) with normal widths (Fig. 9I), some of which aligned with those found in normal owls and others of which were shifted from normal by as much as $150 \mu \mathrm{sec}$ toward left-ear leading (Fig. $9 F$ ). For sites representing space to the far right (visual $\mathrm{RF} \mathrm{az,} \geq \mathrm{R} 25^{\circ}$ ), ITD tuning was roughly normal in shape, best value, and width (Fig. 9D,G,J).

To determine the frequency-specific adjustments that were responsible for these changes in broadband ITD tuning, we compared narrowband and broadband ITD tuning curves for 98 tectal sites in device-reared owls. Using narrowband stimuli with center frequencies of between 3.0 and $9.0 \mathrm{kHz}, 201$ of 261 ITD tuning curves had peaks with best values that were within the $50 \%$ cutoff limits of a site-matched broadband ITD peak. Of the narrowband best ITDs that did not fall within these limits, most (39 of 60) were for stimuli with center frequencies of $\leq 5 \mathrm{kHz}$, suggesting that responses to these lower frequencies could be suppressed by cross-frequency inhibition. However, those that did fall within the limits of a broadband peak were for stimuli that covered the entire range of frequencies tested (although relatively fewer were with stimuli near $6 \mathrm{kHz}$ because, at many sites, responses to those stimuli were too weak to determine tuning) and indicated shifts from normal values, the magnitude of which varied with stimulus frequency (Fig. 10). The correspondence between broadband and narrowband best ITDs indicates that in device-reared owls, these shifts in narrowband best ITDs could account for the shifts in broadband ITD tuning.

Narrowband best ITDs in device-reared owls were shifted from normal in a frequency-dependent manner. Figure $10 \mathrm{~A}$ depicts best ITDs measured with a variety of stimulus frequencies for 98 tectal sites with visual RFs located between $\mathrm{L} 41^{\circ}$ and $\mathrm{R} 51^{\circ}$ az and $-17^{\circ}$ and $+27^{\circ}$ el. As in normal owls, narrowband best ITD varied systematically with visual RF azimuth: linear least-squares fits to best ITDs for narrowband stimuli centered near 4 and 8 $\mathrm{kHz}$, respectively, plotted as functions of visual RF azimuth both had slopes of $\sim 3 \mu \mathrm{sec} /{ }^{\circ}$. Unlike the case in normal owls, however, these narrowband best ITDs in device-reared owls tended to be different for different frequencies at a given tectal location. Indeed, the linear fits to the 4 and $8 \mathrm{kHz}$ data had intercepts of -55 and $+8 \mu \mathrm{sec}$, respectively (Fig. 10B).

These device-induced changes in narrowband ITD tuning reflected the frequency-specific, acoustic effects of the device on sound timing. Figure $10 C$ depicts the shifts in narrowband best ITDs relative to predicted normal values for sites in the tectum with frontally located visual RFs (between $\mathrm{L} 25^{\circ}$ and $\mathrm{R} 25^{\circ}$ az and $-17^{\circ}$ and $27^{\circ} \mathrm{el}$ ). For stimuli centered on between 3 and $5 \mathrm{kHz}$, best ITDs were shifted by $-55 \pm 18 \mu \mathrm{sec}$ (mean $\pm \mathrm{SD} ; n=98$ ). In comparison, the device caused a median shift of $-65 \mu \mathrm{sec}$ (range, -33 to $-91 \mu \mathrm{sec} ; n=5$ ) in the timing of $4 \mathrm{kHz}$, as measured with cochlear microphonics (Gold and Knudsen, 1999). For stimuli near $6 \mathrm{kHz}$, best ITDs, which were difficult to characterize because responses were weak, were shifted by $-33 \pm 44$ $\mu$ sec (mean $\pm \mathrm{SD} ; n=28$ ). In comparison, the device caused a median shift of $-29 \mu \mathrm{sec}$ (range, -12 to $-54 \mu \mathrm{sec}$ ) at $6 \mathrm{kHz}$. For stimuli near $8 \mathrm{kHz}$, the distribution of best ITD shifts was roughly bimodal, because most ITD tuning curves had two peaks separated by $\sim 125 \mu \mathrm{sec}$ within the range of ITDs tested. Because these multiple peaks resulted from the periodic equivalence of interaural phase (125 $\mu \mathrm{sec}$ for $8 \mathrm{kHz}$; Fig. $5 B)$, both modes of the distribution indicated a single, underlying shift of ITD tuning at $8 \mathrm{kHz}$. This shift was estimated by analyzing separately the distributions on either side of $-50 \mu \mathrm{sec}$. This analysis yielded $+15 \pm 25 \mu \mathrm{sec}($ mean $\pm \mathrm{SD} ; n=65)$ for one mode and $-108 \pm$ $25 \mu \mathrm{sec}(n=62)$ for the other. In comparison, the device caused a median shift at $8 \mathrm{kHz}$ of $-6 \mu \mathrm{sec}$ (range, -18 to $+53 \mu \mathrm{sec}$ ) or, equivalently, $-131 \mu \mathrm{sec}$.

These device-induced shifts in best ITD relative to predicted 

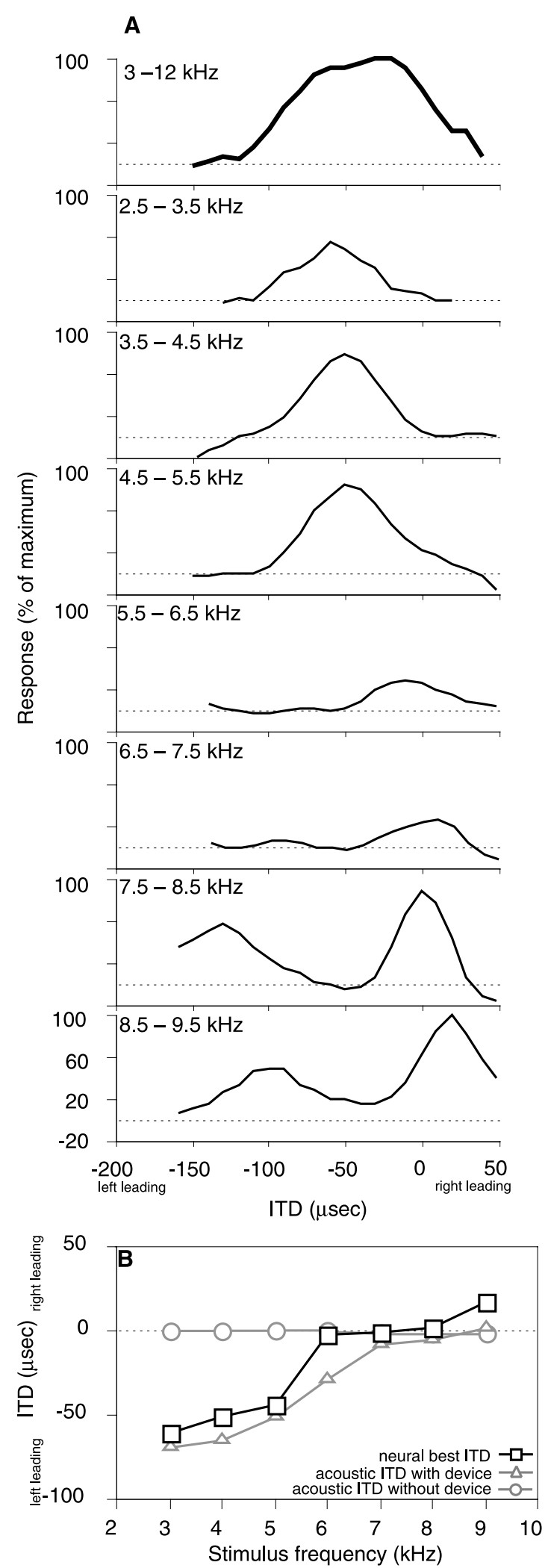

Figure 8. ITD tuning at a tectal site in a device-reared owl. The visual $\mathrm{RF}$ at this site was centered at $0^{\circ}$ az, $+8^{\circ}$ el. $A$, ITD tuning curves obtained using broadband (3-12 kHz, top curve) or narrowband (the range of stimulus frequencies used is shown for each curve) stimuli. Broadband responses were normalized relative to the maximum response elicited with the broadband stimulus. Tuning curves for all narrowband stimuli were normalized relative to the maximum response elicited with any narrowband stimulus. In some cases, a second, smaller peak appeared normal values varied systematically across the space map. In general, shifts were larger for neurons in the right tectum, representing the left hemifield (i.e., space contralateral to the device), than for those in the left tectum, representing the right hemifield. For narrowband stimuli near $4 \mathrm{kHz}$ (Fig. 11A), the shift was $-58 \pm 14 \mu \mathrm{sec}$ (mean $\pm \mathrm{SD}$ ) in the right tectum and $-47 \pm 19$ $\mu \mathrm{sec}$ in the left tectum; this difference was significant (unpaired $t$ test; $p<0.01)$. For narrowband stimuli near $6 \mathrm{kHz}$ (Fig. 11B), the best ITDs relative to expected normal values tended to be more left-ear leading in the right tectum than in the left tectum, but these measurements were sparse, and this difference was not significant $(-35 \pm 41 \mu \mathrm{sec}$ vs $-20 \pm 42 \mu \mathrm{sec} ; p=0.35)$. For narrowband stimuli near $8 \mathrm{kHz}$ (Fig. $11 C$ ), regression lines relating best ITD shift with visual RF azimuth were significant (ANOVA; $p<0.001$ ) for both modes of the distribution of best ITDs, indicating shifts that were more right-ear leading for sites with visual RFs to the left. For the mode of the distribution nearest to predicted normal values, this regression indicates larger shifts in the right tectum (which represents space to the left) than in the left tectum.

Device rearing affected not only narrowband best ITDs but, at some sites, the suppression of interaural phase-equivalent peaks, as well. In normal owls, ITD tuning curves for narrowband (1 $\mathrm{kHz}$ bandwidth) stimuli near $8 \mathrm{kHz}$ had interaural phaseequivalent peaks at their predicted locations (separated by $\sim 125$ $\mu \mathrm{sec})$. In contrast, some $8 \mathrm{kHz}$ ITD tuning curves in devicereared owls had only a single peak within the same range of ITDs, because of the suppression of one or more of the interaural phase-equivalent peaks by interactions across frequency channels (Takahashi and Konishi, 1986) that were within the passband of the stimulus (Fig. 5A). This suppression was not evident for most sites (Fig. 11C; circles represent curves with multiple peaks, triangles represent curves with a single peak). When present, however, the characteristics of this suppression varied as a function of location in the space map and, therefore, as a function of the magnitude of shift. For the curves measured at sites representing space to the far left (in the right tectum, where the biggest shifts toward right-ear leading were found), for example, the peak nearest the normal predicted value was often suppressed, but the peak one period away was not (Fig. 11C, filled triangles). In contrast, for the curves measured at sites representing space to the far right (in the left tectum), the relatively unshifted peak nearest the normal expected value was present, but responses to the ITD one period away were usually suppressed (Fig. 11C, open triangles).

\section{ILD tuning in normal owls}

In normal owls, acoustic ILD varies with both sound source location (Olsen et al., 1989) and frequency (Knudsen et al., 1991). We found that, accordingly, best ILD in the optic tectum varied with both visual RF elevation, which has been reported previously (Olsen et al., 1989), and stimulus frequency, which has not been reported previously.

$\leftarrow$

when using a narrowband stimulus (e.g., stimulus $=7.5-8.5 \mathrm{kHz}$ ). In each case, the location of the second peak matched the expected location of an interaural phase equivalent peak for the center frequency of the given range. $B$, Best values from the tuning curves in $A$ for narrowband stimuli plotted as a function of the center frequency (squares), along with the frequency-specific, average acoustic ITDs experienced by normal owls (circles) and by owls wearing the acoustic device (triangles) for sound sources located in the center of the visual RF of this site. 

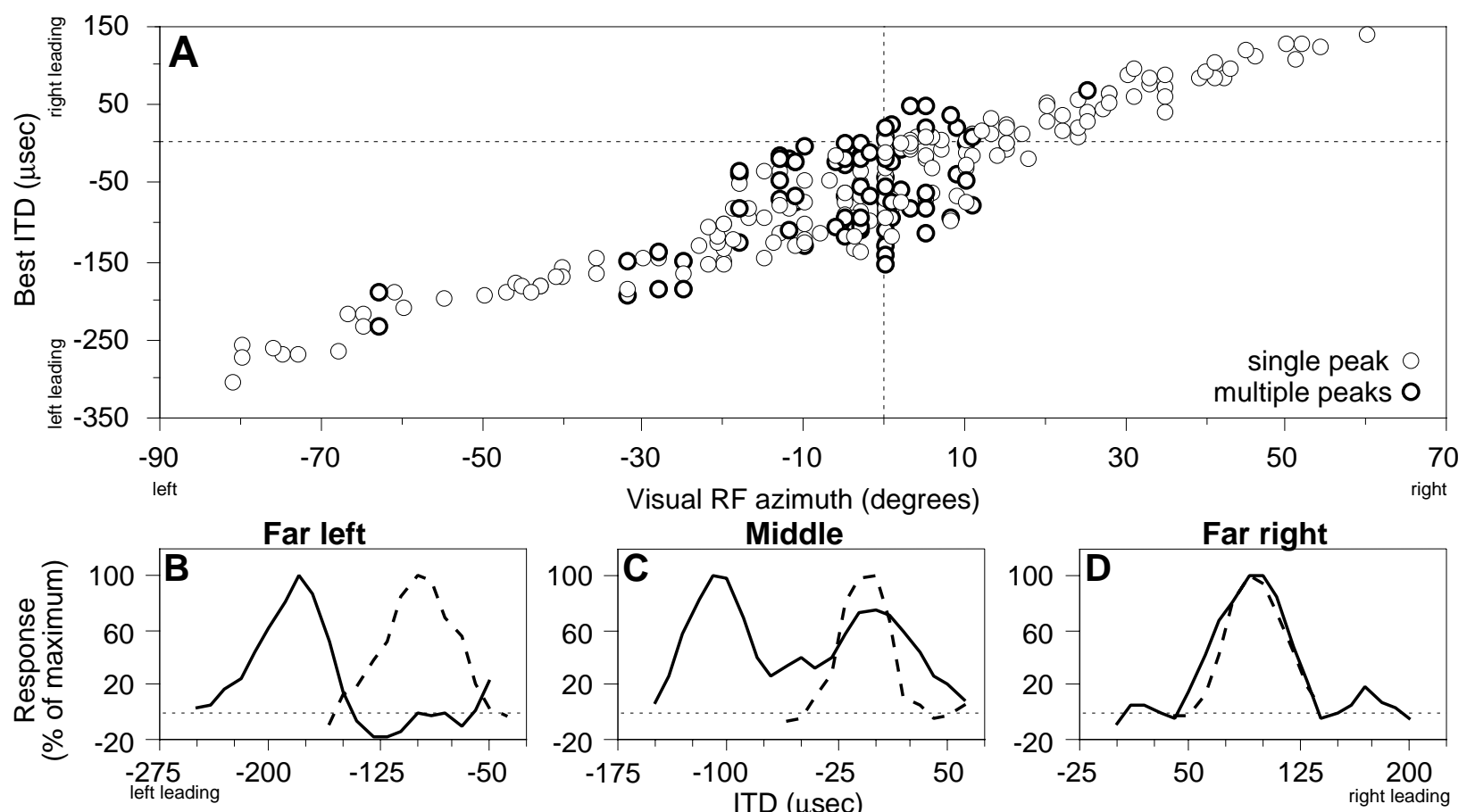

Visual RF azimuth (degrees) Middle
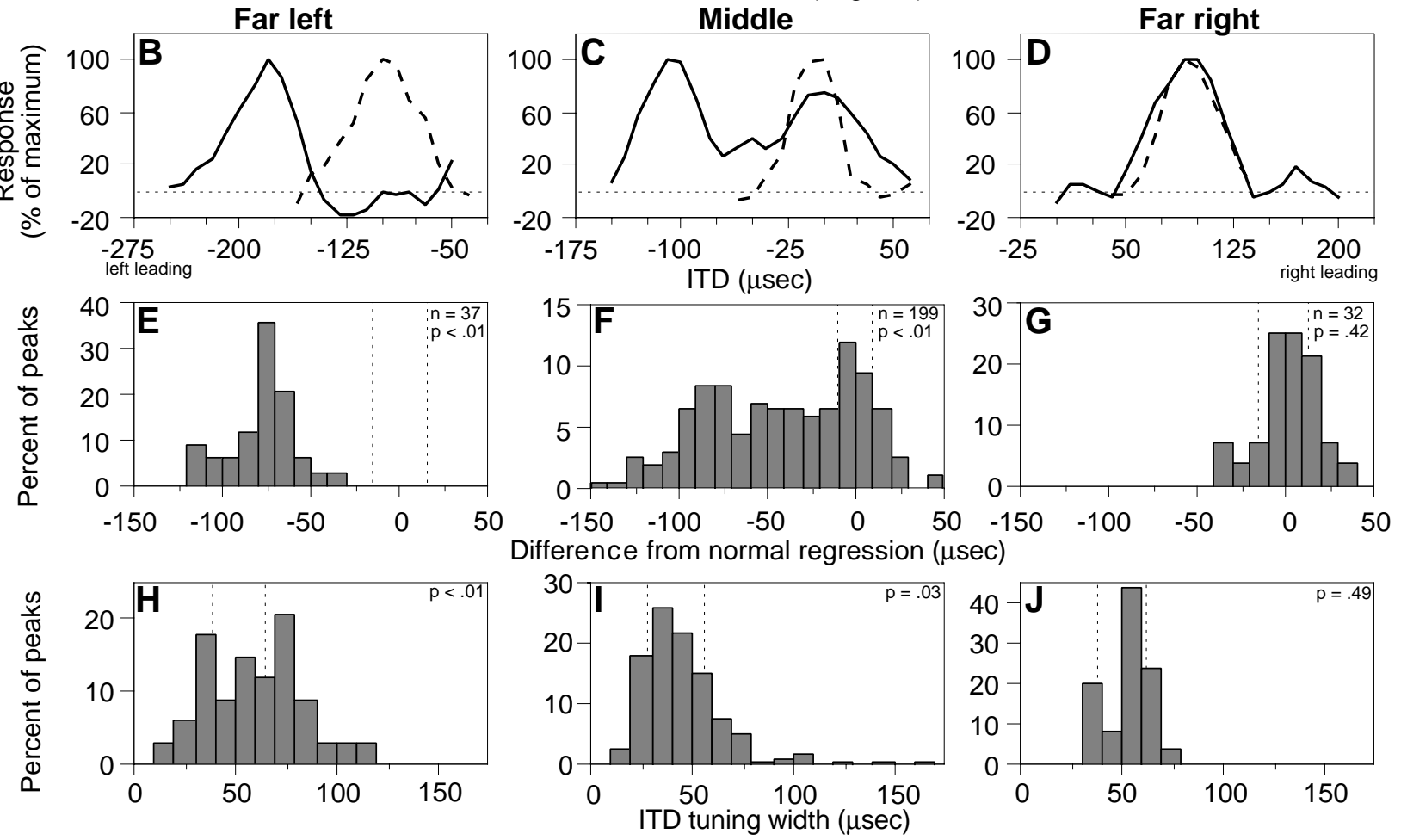

Figure 9. Summary of broadband ITD tuning of tectal neurons in device-reared owls. $A$, Best ITD as a function of visual RF azimuth from 219 sites in eight device-reared owls. The linear fit from previously published normal data are plotted for comparison (dashed line; Brainard and Knudsen, 1993). At 38 sites, ITD tuning curves had multiple peaks, which are shown in bold. ITD tuning fell into three categories that roughly corresponded to regions representing space to the far left (visual RF az, $\geq \mathrm{L} 25^{\circ}$; panels $B, E$, and $H$ ), directly ahead (visual RF az between $\mathrm{L} 25^{\circ}$ and $\mathrm{R} 25^{\circ}$; panels $C$, $F$, and $I$ ), and to the far right (visual RF az, $\geq \mathrm{R} 25^{\circ}$; panels $D, G$, and $J$ ). $B-D$, Examples of ITD tuning curves from device-reared (solid) and normal (dashed) owls. In each panel, curves were measured at sites with matching visual RF locations (visual RF azimuths were $\mathrm{L} 40^{\circ}$, L2 ${ }^{\circ}$ and $\mathrm{R} 41^{\circ}$, respectively). Distributions of the differences between best ITDs and the normal regression $(E-G)$ and of ITD tuning widths $(H-J)$ are shown for each region: far left , mean $\pm \mathrm{SD}$ difference $=-77 \pm 18 \mu \mathrm{sec}(E)$, mean $\pm \mathrm{SD}$ width $=60 \pm 23 \mu \mathrm{sec}(H)$. Middle, Difference $=-42 \pm 45 \mu \mathrm{sec}(F)$, width $=46 \pm 22$ $\mu \sec (I)$. Far right, Difference $=2 \pm 16 \mu \mathrm{sec}(G)$, width $=53 \pm 11 \mu \mathrm{sec}(J)$. The dashed lines in $E-J$ represent mean \pm SD values in normal owls. $p$ values from unpaired $t$ tests comparing the given distribution of data with comparable data from normal owls are shown in $E-J$.

Tectal units were tuned for broadband and narrowband ILD. Figure 12 shows broadband and narrowband ILD tuning for a site with a visual $\mathrm{RF}$ at $\mathrm{R} 5^{\circ} \mathrm{az},+10^{\circ} \mathrm{el}$ in a normal owl. For this site, the broadband ILD tuning curve had a peak centered at $5.8 \mathrm{~dB}$ with a width of $9.2 \mathrm{~dB}$. Narrowband ILD tuning curves had broader peaks, with best values of $2.4 \mathrm{~dB}$ at $4 \mathrm{kHz}$ and $\sim 6-7 \mathrm{~dB}$ at higher frequencies. This relationship between best ILD and frequency matched the pattern of acoustic ILDs produced by a source located in the visual RF of the site (Fig. 12B).
For 23 sites with visual RFs between $\mathrm{L} 20^{\circ}$ and $\mathrm{R} 17^{\circ}$ az and $-10^{\circ}$ and $+15^{\circ} \mathrm{el}$, tectal neurons were tuned for broadband ILD (width, $12.0 \pm 4.3 \mathrm{~dB}$; mean $\pm \mathrm{SD}$ ), and best ILD varied systematically with visual RF elevation, as reported previously (Olsen et al., 1989). Moreover, as depicted in Figure 13, $A$ and $B$, the frequency-specific best ILDs at a given tectal site were similar to the acoustic ILDs produced by a source located in the visual $\mathrm{RF}$ of the site. In many cases, these narrowband best ILDs varied with frequency: for individual sites, best ILDs for 4 and 8 

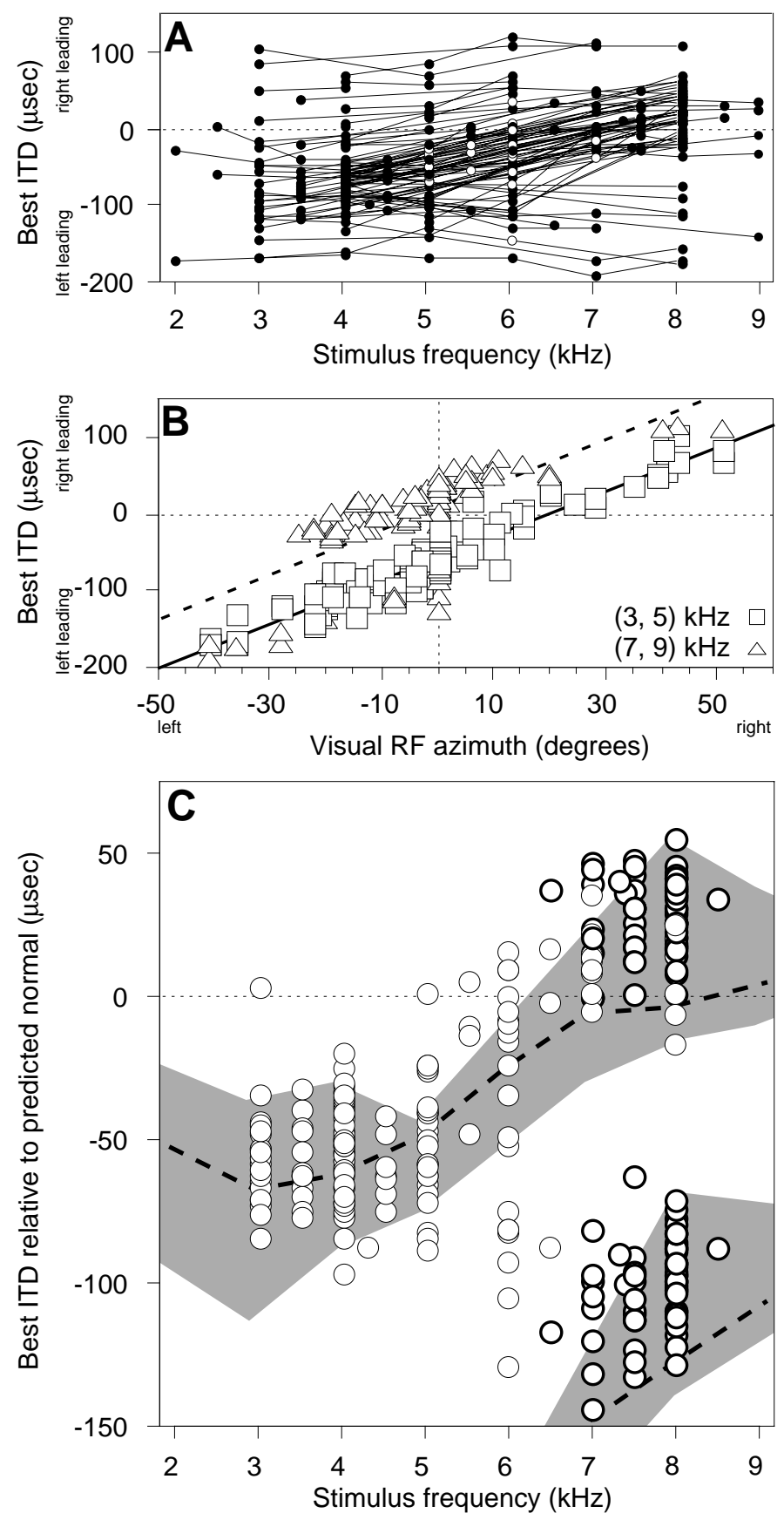

Figure 10. Summary of narrowband ITD tuning of tectal neurons in device-reared owls. Data are from 98 sites with visual RFs between $\mathrm{L} 41^{\circ}$ and $\mathrm{R} 51^{\circ}$ az and $-17^{\circ}$ and $+27^{\circ}$ el. $A$, Best ITD as a function of center stimulus frequency. Each line connects points representing measurements taken at a single recording site. The open circles represent frequencies that did not elicit strong enough responses to measure ITD tuning at the given sites. $B$, Best ITDs for low-frequency $(3,5 \mathrm{kHz})$ or high-frequency $(7,9$ $\mathrm{kHz}$ ) stimuli as functions of visual RF azimuth. Linear fits are shown for the low- (solid line; $y=2.9 \mathrm{x}-55.2 ; r^{2}=0.91$ ) and high- (dashed line; $y=$ $\left.3.0 \mathrm{x}-8.0 ; r^{2}=0.54\right)$ frequency data, both of which were highly significant (ANOVA, $p<0.001$ ). $C$, Best ITD relative to the acoustic ITD produced by a source located at the visual RF of the site as a function of the center frequency of the narrowband stimulus. Data are from sites with visual RFs between $\mathrm{L} 25^{\circ}$ and $\mathrm{R} 25^{\circ}$ az. For ITD tuning curves with two peaks within the range of ITDs tested, both peaks are shown in bold. Dashed lines and shaded regions represent the median values and the ranges, respectively, of phase-equivalent shifts in the timing of sound reaching the right eardrum caused by insertion of the acoustic filtering device (cochlear microphonic measurements from five owls; Gold and Knudsen, 1999).

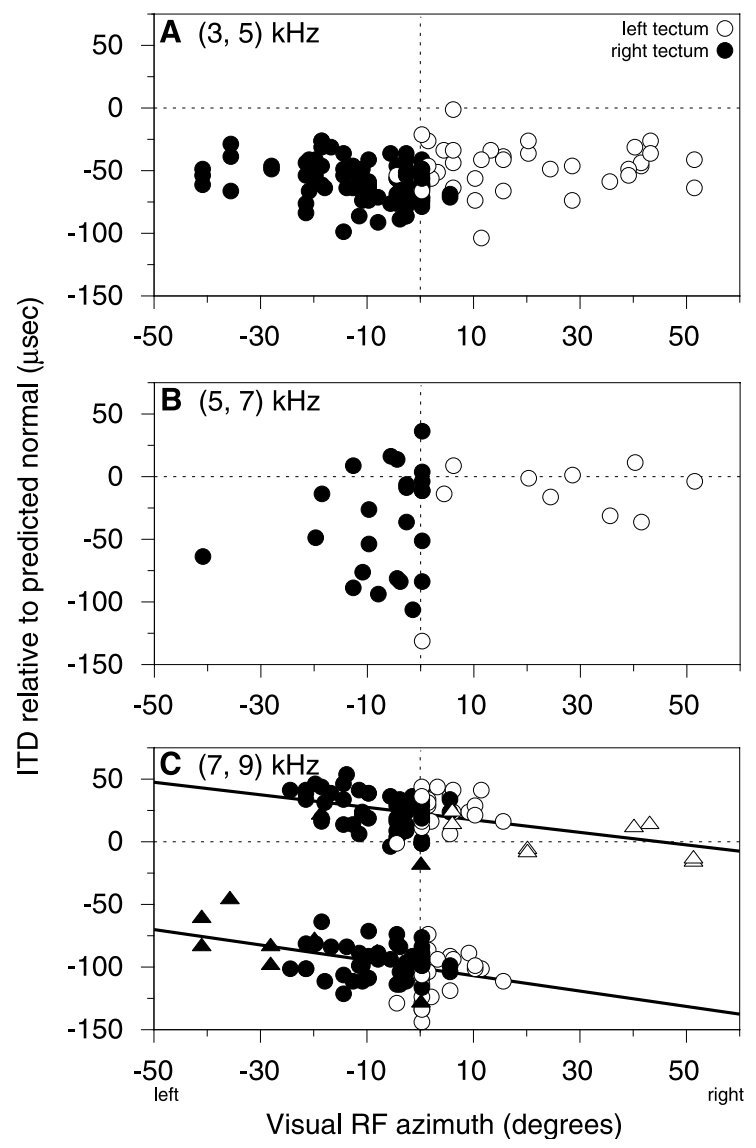

Figure 11. Device-induced shifts in frequency-specific ITD tuning as a function of location in the tectal map. In each panel, best ITD relative to the predicted normal value (based on visual RF location) for a narrowband stimulus near 4,6 , or $8 \mathrm{kHz}$ is plotted as a function of visual RF azimuth. Open and closed symbols represent data taken from the left and right tecta, respectively. $A$, ITD shifts for $6 \mathrm{kHz}$ stimuli did not significantly regress as a function of visual RF azimuth $\left(r^{2}=0.17\right.$; ANOVA, $p=0.07)$, although ITD shifts measured in the left $(-47 \pm 19 \mu \mathrm{sec}$; mean $\pm \mathrm{SD})$ and right $(-58 \pm 14 \mu \mathrm{sec})$ tecta differed significantly (unpaired $t$ test, $p<0.001$ ). $B$, ITD shifts for $6 \mathrm{kHz}$ stimuli did not significantly regress as a function of visual RF azimuth $\left(r^{2}=0.09\right.$; ANOVA, $p=0.08)$. $C$, The solid lines are linear least-squares fits to the two distributions $\left(y=-0.5 \mathrm{x}+22.5 ; r^{2}=0.20\right.$ above and $y=-0.6 \mathrm{x}-$ 100.8; $r^{2}=0.20$ below; ANOVA, $p<0.001$ in both cases). Triangles indicate best values from tuning curves with a single peak within the range of ITDs tested.

$\mathrm{kHz}$ stimuli differed by, on average, $8.9 \pm 4.3 \mathrm{~dB}$ (mean $\pm \mathrm{SD}$; $n=11)$.

\section{ILD tuning in device-reared owls}

ILD tuning was abnormal in device-reared owls, with broadband and narrowband best ILDs typically shifted from normal values in the adaptive direction. In most cases, however, these shifts in ILD tuning were substantially smaller than expected based on the acoustic effects of the device. Figure 14 shows an example of broadband and narrowband ILD tuning for a site with a visual RF at $\mathrm{L} 11^{\circ} \mathrm{az},+11^{\circ} \mathrm{el}$. For this site, the broadband ILD tuning curve had a best value of $0 \mathrm{~dB}$ and a width of $6.9 \mathrm{~dB}$. Narrowband ILD tuning curves were broader and had best values that were close to predicted normal values for low frequencies but were closer to the acoustic values experienced with the device in place for higher frequencies. For example, for the $4 \mathrm{kHz}$ stimulus, the best ILD was $-3 \mathrm{~dB}$, which was slightly more left-ear greater than the 

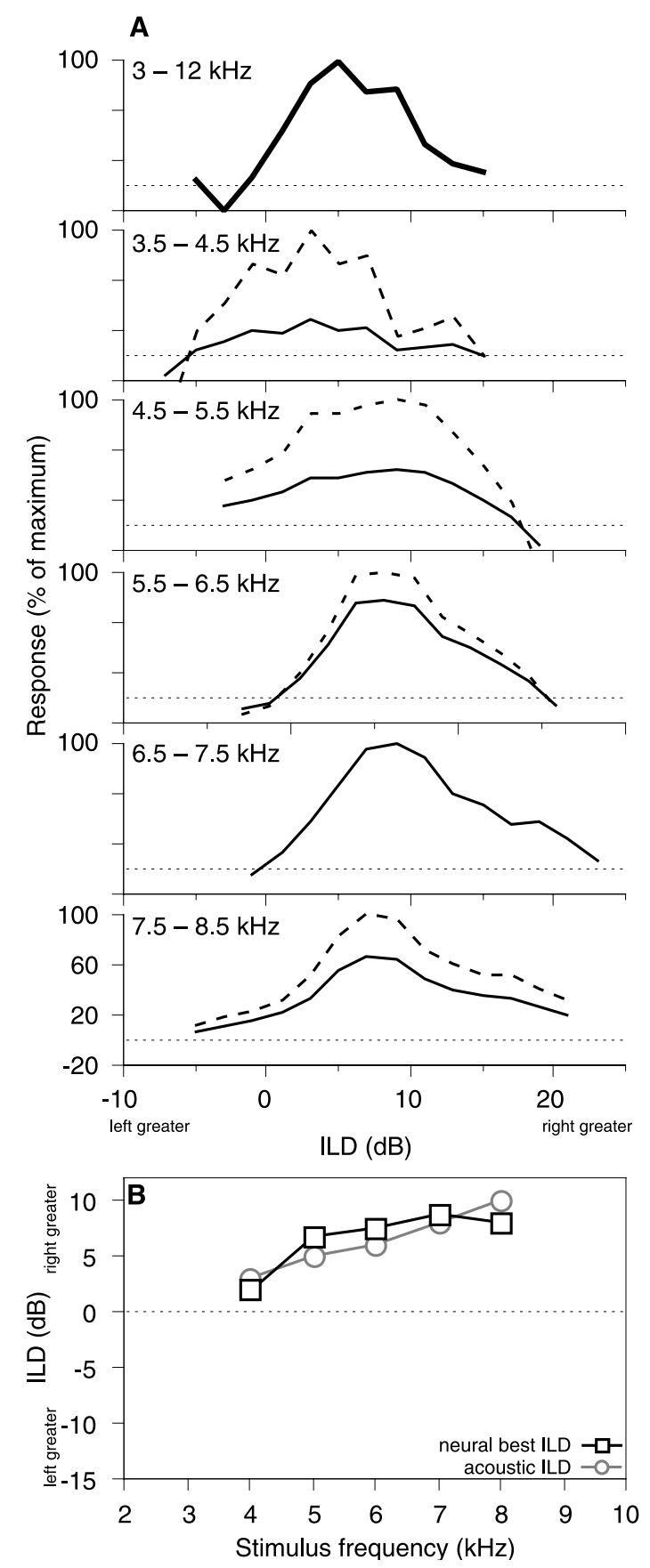

Figure 12. ILD tuning at a tectal site in a normal owl. The visual RF at this site was centered at $\mathrm{R} 5^{\circ}$ az, $+10^{\circ}$ el. $A$, ILD tuning curves for a broadband stimulus (3-12 kHz, top curve) and for five different narrowband stimuli (the range of stimulus frequencies used is shown for each curve). Broadband responses were normalized relative to the maximum response elicited with the broadband stimulus. Narrowband responses were normalized relative to the maximum response elicited with the given stimulus (dashed curves) or with any narrowband stimulus (solid curves). $B$, Best values from the curves in $A$, using narrowband stimuli (squares), along with the average acoustic ILDs experienced by normal owls for a source located in the visual RF of the site (circles), as a function of the center frequency of the narrowband stimulus.

acoustic ILD experienced by normal owls for a source located at the visual RF but was not completely shifted to the value experienced by owls wearing the device (Fig. 14B). For the $6 \mathrm{kHz}$ stimulus, responses were weak but ILD-tuned, with a relatively
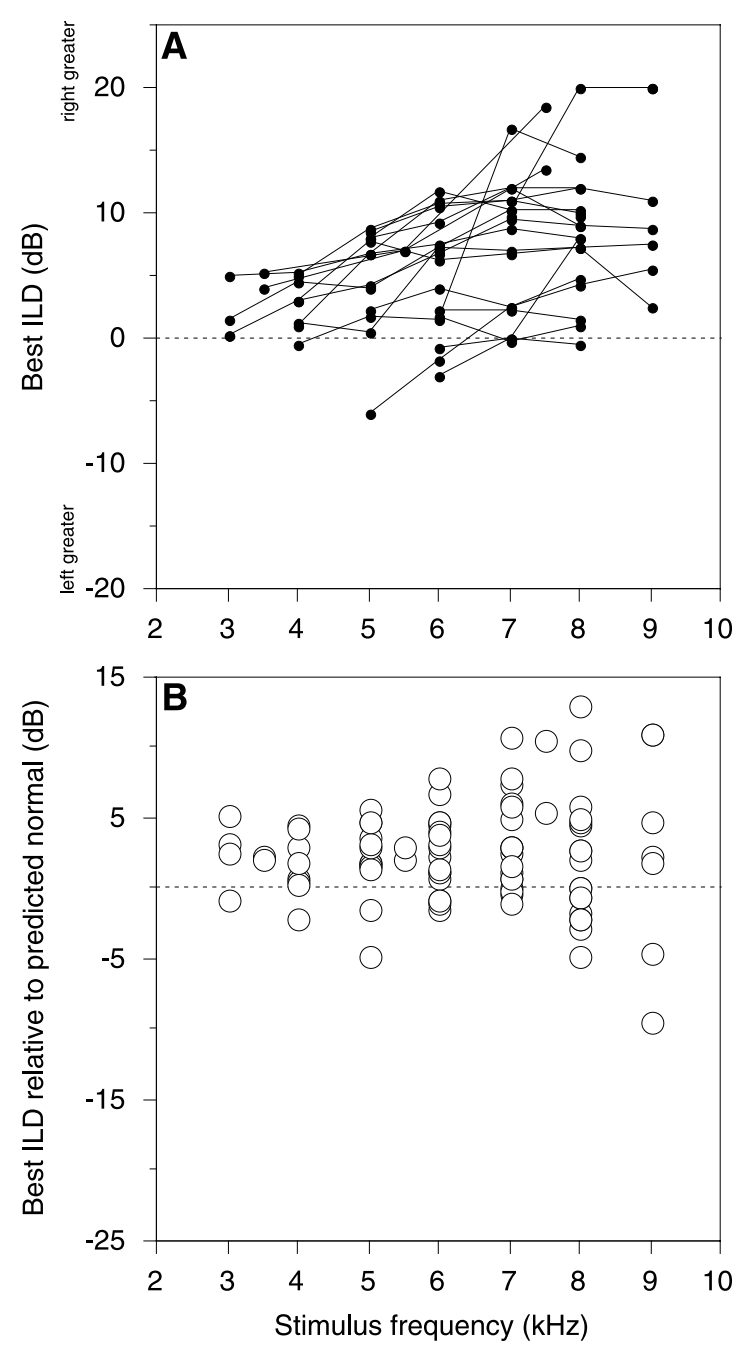

Figure 13. Summary of narrowband ILD tuning of tectal neurons in normal owls. $A$, Best ILD as a function of center stimulus frequency. Each line connects points representing measurements taken at a single recording site. $B$, Best ILDs from $A$ relative to the predicted normal best ILDs, plotted as a function of stimulus frequency; the two were not correlated (ANOVA, $p=0.85$ ).

unshifted best value. For the $8 \mathrm{kHz}$ stimulus, responses were very strong, and the best ILD was close to the acoustic ILD experienced with the device in place, which was shifted from the normal predicted value by $\sim 15 \mathrm{~dB}$ toward left-ear greater (Fig. 14B).

For sites with visual RFs between $\mathrm{L} 25^{\circ}$ and $\mathrm{R} 25^{\circ}$ az in devicereared owls, broadband ILD tuning curves had slightly but significantly narrower peak widths than comparable curves in normal owls $(8.9 \pm 3.6 \mathrm{~dB}$ vs $12.0 \pm 4.3 \mathrm{~dB}$ in normal owls; unpaired $t$ test; $p<0.01$; Fig. $15 C$ ). Like in normal owls, these peaks had best values that varied as a function of visual RF elevation (Fig. 15A). For nearly all sites, however, best ILD was shifted toward left-ear greater values relative to the control regression ( shift $=-6.6 \pm$ $4.4 \mathrm{~dB}$; mean $\pm \mathrm{SD}$; Fig. $15 A, B)$. For sites with broadband ITD tuning curves with more than one peak, best ILDs measured using the best ITD from each peak differed by only $2.2 \pm 1.4 \mathrm{~dB}$ (mean $\pm \mathrm{SD}$ ); in those cases, the mean best ILD is shown. Moreover, the shifts in best ILD relative to predicted normal values varied weakly with location in the space map. In general, 
A
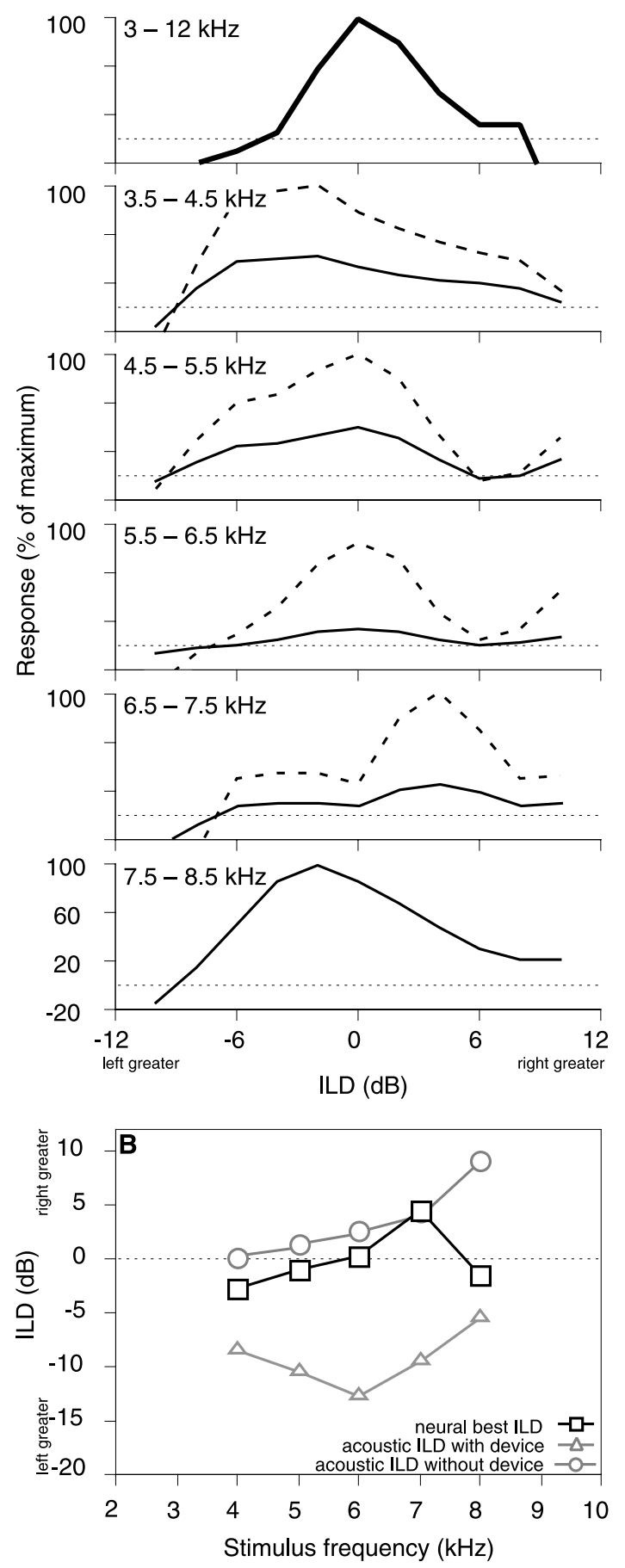

Figure 14. ILD tuning at a tectal site in a device-reared owl. The visual $\mathrm{RF}$ at this site was centered at $\mathrm{L} 11^{\circ} \mathrm{az},+11^{\circ} \mathrm{el} . A$, ILD tuning curves for a broadband stimulus (3-12 kHz, top curve) and for five different narrowband stimuli (the range of stimulus frequencies used is shown for each curve). Broadband responses were normalized relative to the maximum response elicited with the broadband stimulus. Narrowband responses were normalized relative to the maximum response elicited with the given stimulus (dashed curves) or with any narrowband stimulus (solid curves). $B$, Best values from the curves in $A$, using narrowband stimuli (squares), along with the average acoustic ILDs experienced by normal owls (circles) and owls wearing the acoustic device (triangles) for a source located in the visual RF of this site, as a function of the center frequency of the narrowband stimulus.
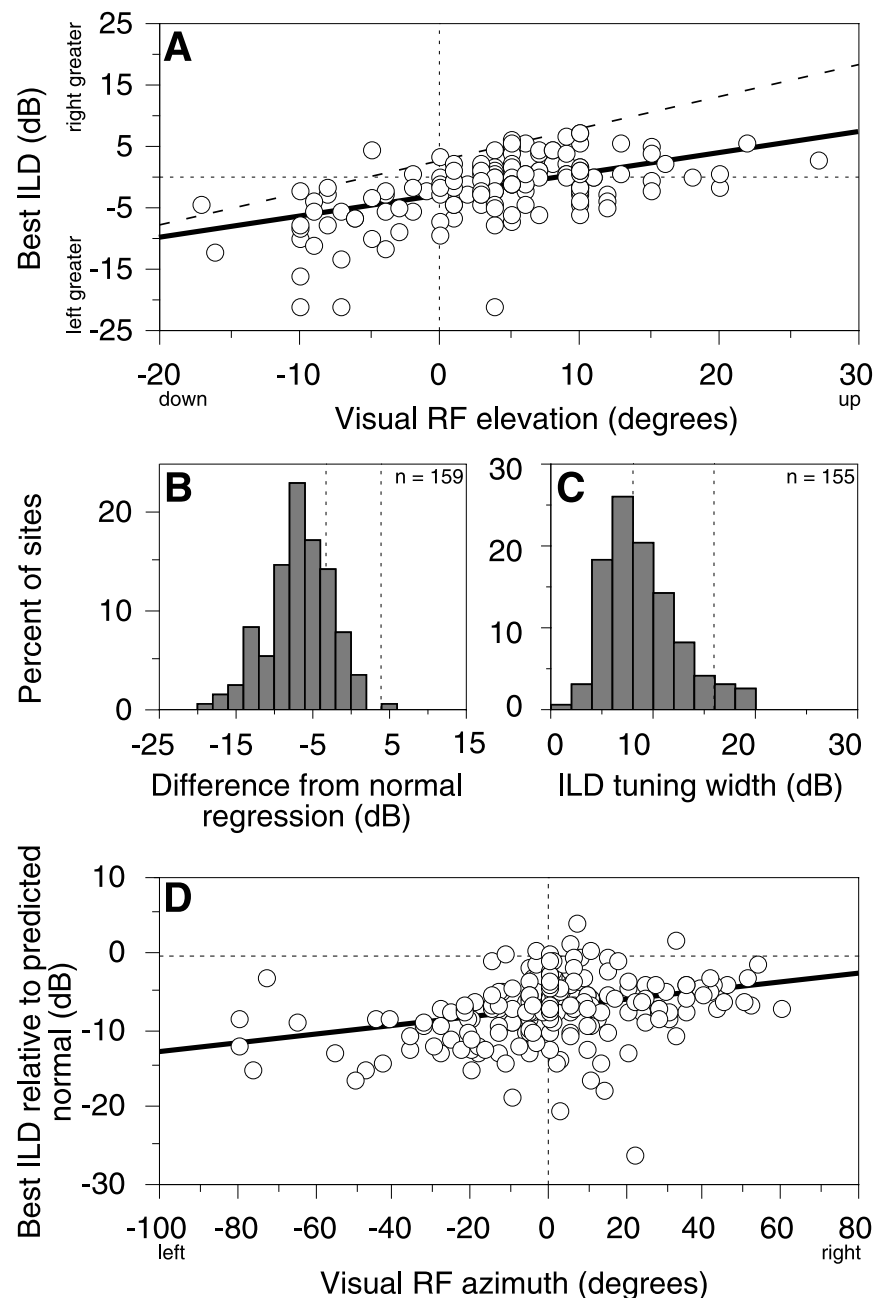

Figure 15. Summary of broadband ILD tuning of tectal neurons in device-reared owls. $A$, Best ILDs for broadband stimuli as a function of visual RF elevation from sites with visual RFs between $\mathrm{L} 25^{\circ}$ and $\mathrm{R} 25^{\circ}$ az. The dashed line is the linear least-squares regression from previously published normal data (Olsen et al., 1989). The solid line is a linear fit to the data from device-reared owls $\left(y=0.3 \mathrm{x}-3.2 ; r^{2}=0.31\right.$; ANOVA, $p<$ 0.001). $B$, Distribution of best ILDs relative to the normal regression; mean $\pm \mathrm{SD}=-6.6 \pm 4.4 \mathrm{~dB}$. $C$, ILD tuning widths for the data in $A$; mean $\pm \mathrm{SD}=8.6 \pm 3.3 \mathrm{~dB}$. The dashed line indicates the mean ILD tuning width measured in normal owls. $D$, Best ILDs relative to the normal regression as a function of visual RF azimuth. The solid line is a linear fit to the data that indicated a weak dependence of best ILD shift on visual RF azimuth $\left(y=0.06 \mathrm{x}-6.8 ; r^{2}=0.09\right.$; ANOVA, $\left.p<0.001\right)$.

shifts were slightly smaller for sites representing space downward (Fig. 15A, compare solid and dashed lines) and to the right (Fig. $15 D)$. These smaller shifts are consistent with the directiondependent effects of the device on sound level, which were smaller at all frequencies for sound sources located to the right (Gold and Knudsen, 1999).

Narrowband ILD tuning was also abnormal in device-reared owls, although in many cases the device-induced changes did not match the acoustic effects of the device (data from 65 sites are summarized in Fig. 16). For narrowband stimuli with center frequencies in the range of $3-5 \mathrm{kHz}$, best ILDs were shifted significantly (unpaired $t$ test; $p<0.01$ ) from normal predicted values by $-2.8 \pm 2.7 \mathrm{~dB}(n=62)$, but these best ILDs were closer to the average acoustic values experienced without the device 

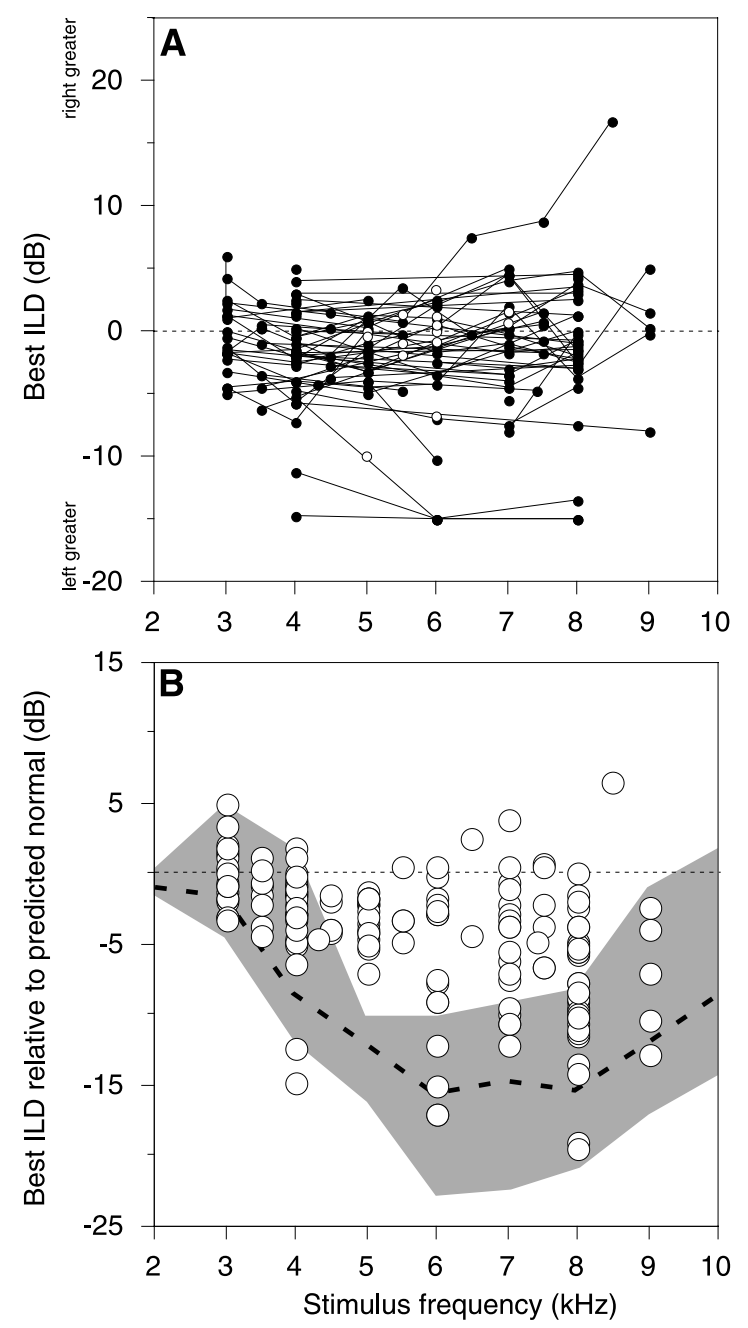

Figure 16. Summary of narrowband ILD tuning of tectal neurons in device-reared owls. Data are from sites with visual RFs between $\mathrm{L} 25^{\circ}$ and $\mathrm{R} 25^{\circ}$ az. $A$, Best ILD as a function of the center frequency of the narrowband stimulus. Each line connects points representing measurements taken at a single recording site. The open circles represent frequencies that did not elicit strong enough responses to measure ILD tuning at the given sites. $B$, Best ILD relative to the normal acoustic ILD, plotted as a function of stimulus frequency. The dashed line and shaded region represent the median values and the ranges, respectively, of shifts in the level of sound reaching the right eardrum caused by insertion of the acoustic filtering device (cochlear microphonic measurements from four owls; Gold and Knudsen, 1999).

than with the device in place. For narrowband stimuli with center frequencies in the range of $5-7 \mathrm{kHz}$, measurements were variable and sparse because of the lack of responsiveness at these frequencies in device-reared owls. When measurable, best ILDs were shifted significantly from normal predicted values by $-5.3 \pm 5.1$ $\mathrm{dB}(n=22)$, but these shifts were incomplete, being closer to the average acoustic values experienced without the device than with the device in place. For narrowband stimuli with center frequencies in the range of $7-9 \mathrm{kHz}$, best ILDs were also highly variable but, on average, were shifted significantly from normal predicted values by $-7.5 \pm 4.6 \mathrm{~dB}(n=41)$. These shifts were, again, incomplete, being approximately halfway between the average acoustic values experienced with and without the device in place.

\section{DISCUSSION}

The present results demonstrate that early experience can alter tectal unit tuning for binaural localization cues in a frequencydependent manner. In normal owls, such adjustments allow tectal neurons to match their response properties to the cues produced by the acoustic filtering effects of the head and ears (Figs. 6, 12). In animals that experience hearing impairments, this capacity appears to have limits but helps tectal neurons to interpret abnormal cue values correctly to encode the true location of an auditory stimulus. The following sections discuss how these results provide insights into principles of information processing and adaptive plasticity in the auditory localization pathway.

\section{Plasticity of frequency tuning}

The broad frequency tuning of tectal neurons enables them to integrate cues across frequency channels, necessary to eliminate spatial ambiguities inherent to individual cues (Brainard et al., 1992); to detect spectral cues (Carlile and King, 1994); and to encode the locations of a wide range of acoustic stimuli, regardless of the frequency content of the stimulus. We found that this frequency tuning can be altered dramatically by early auditory experience: for the majority of tectal neurons, device rearing substantially reduced normally robust responses to stimuli near 6 kHz (Figs. 1-4).

A possible explanation for this effect is that the device caused pathology resulting in sensorineural hearing loss to frequencies near $6 \mathrm{kHz}$. However, cochlear microphonics measured in devicereared owls with the device removed did not reveal any reduction in the magnitude of responses to $6 \mathrm{kHz}$ stimuli relative to other frequencies. In addition, recordings in the central nucleus of the inferior colliculus of these same owls indicated normal thresholds and frequency responses for all stimuli between 4 and $8 \mathrm{kHz}$ (J. I. Gold and E. I. Knudsen, unpublished observations). Thus, the device altered the frequency tuning of tectal neurons without affecting the sensitivity of the ear to $6 \mathrm{kHz}$ sounds.

The decrease in responses to $6 \mathrm{kHz}$ stimuli might be accounted for by two effects of the acoustic device. First, the device severely altered sound timing and level at $6 \mathrm{kHz}$, with median shifts of -29 $\mu \mathrm{sec}$ and $-16 \mathrm{~dB}$, respectively. In contrast, lower frequencies had larger time shifts but much less attenuation, and higher frequencies had smaller time shifts and slightly less attenuation. Thus, 6 $\mathrm{kHz}$ stimuli were consistently more attenuated than other frequencies and rarely produced combinations of ITD and ILD values that were within the normal range. For example, with the device in place, the $6 \mathrm{kHz}$ sound level was always greater in the left (open) ear, regardless of sound source location. Consequently, tectal sites tuned to right-ear greater ILDs at $6 \mathrm{kHz}$ (sites with visual RFs above $\sim 0^{\circ}$ elevation; Brainard et al., 1992) could never be activated strongly by $6 \mathrm{kHz}$ stimuli. For these reasons, tectal neurons were relatively deprived of activity driven by $6 \mathrm{kHz}$ stimuli.

Second, the effects of the device were most frequencydependent near $6 \mathrm{kHz}$, particularly for ITD, which changed by $\sim 50 \mu \mathrm{sec}$ between 5 and $7 \mathrm{kHz}$ (Fig. 10C). This frequencydependent variation must have blurred the spatial information around $6 \mathrm{kHz}$, because even neurons that are narrowly tuned for frequency integrate information across a finite bandwidth. Because of this integration, neurons involved in the binaural comparison of timing and level would derive different values of ITD and ILD, respectively, for a stimulus at the same location in space, depending on the amplitude spectrum near $6 \mathrm{kHz}$. Thus, 
the ability of $6 \mathrm{kHz}$ stimuli to drive tectal neurons would be further compromised.

These effects may have resulted in the weak responses and variable shifts in ITD and ILD tuning for $6 \mathrm{kHz}$ stimuli (Figs. $11 B, 16 B)$. This interpretation implies that the frequency tuning of tectal neurons is shaped by an activity-dependent process that regulates the strength of responses to particular frequencies according to the degree to which each frequency contributes to postsynaptic discharges. Such a mechanism could be responsible for the normal topographic variation in frequency tuning across the tectum that matches the filtering properties of the external ears (Knudsen, 1984; Keller et al., 1998).

\section{Plasticity of ITD tuning}

Device-induced changes in tectal unit ITD tuning demonstrated that the auditory system interprets and represents ITDs in a frequency-specific and adaptive manner. In regions of the optic tectum representing frontal space in device-reared owls, best ITDs were shifted from normal by averages of $55 \mu \mathrm{sec}$ toward open-ear leading for $4 \mathrm{kHz}$ stimuli and $15 \mu \mathrm{sec}$ in the opposite direction for $8 \mathrm{kHz}$ stimuli. These changes were similar to the frequency-specific, acoustic effects of the device on sound timing for frontally located sources (Fig. 10C). In other regions of the space map, changes in ITD tuning varied in magnitude and tended to be smaller in the left tectum (Figs. 9A, 11). A similar, unexplained phenomenon was found in owls raised with one ear occluded, in which adjustments in ITD and ILD tuning were larger and more systematic in the optic tectum ipsilateral to the occluded ear (Mogdans and Knudsen, 1992). In device-reared owls, this variability across the space map may reflect, in part, direction-dependent acoustic effects of the device. Indeed, a previous study demonstrated that device-induced adjustments throughout the space map were adaptive in that, with the device in place, they tended to restore the alignment of frequencyspecific auditory RFs with the visual RFs at all sites (Gold and Knudsen, 1999).

The frequency-dependent variation in ITD tuning found in device-reared owls means that tectal sites do not necessarily exhibit a "characteristic delay", which is, by definition, independent of frequency (Rose et al., 1966; Yin and Kuwada, 1983). Conversely, ITD-sensitive neurons in the tonotopic auditory pathway leading to the optic tectum do exhibit a characteristic delay (Wagner et al., 1987). The present results indicate that in the process of translating ITD values into a space code, input from neurons in the tonotopic pathway that represent different characteristic delays can be combined to reflect frequency-specific delays experienced by the individual. This type of frequencydependent adaptability is useful even for normal owls, because the acoustic ITDs that they experience vary strongly with frequency for sources located in many regions of space (Knudsen et al., 1991).

\section{Plasticity of ILD tuning}

In all species, the head and ears affect sound level in a frequencydependent manner, producing ILDs that vary markedly with frequency. Therefore, to extract the most information possible from ILD cues, the auditory system must interpret ILD on a frequency-by-frequency basis. Our data suggest only a partial adherence to this principle. In normal owls, best ILDs varied somewhat with stimulus frequency at many sites (differing by $\sim 9$ $\mathrm{dB}$ for 4 and $8 \mathrm{kHz}$ stimuli, for example). In device-reared owls, best ILDs for frequencies near 4,6 , and $8 \mathrm{kHz}$ were shifted from normal in the adaptive direction by averages of, respectively, 3,5 , and $8 \mathrm{~dB}$ (Fig. 16). These changes in ILD tuning were, however, highly variable and, in many cases, did not match the acoustic effects of the device.

The apparent incompleteness of the adaptation of ILD tuning may reflect, in part, the large individual variability in the acoustic effects of the device. For frequencies around 3 and $4 \mathrm{kHz}$, for example, shifts in best ILD fell short of the median acoustic effects of the device in 70 of 76 measurements. However, they fell within the ranges of those effects in all but two cases (Fig. 16B). In addition, because the acoustic effects of the device were measured at $1 \mathrm{kHz}$ intervals, it is possible that spectral notches or changes in the spatial fine-structure of the ILD cues that were induced by the device were not adequately assessed.

The incomplete adjustments in narrowband best ILDs may have alternative explanations, as well. For example, for frequencies near $6 \mathrm{kHz}$, the lack of device-induced adjustment in ILD tuning may have resulted from a deprivation effect caused by the device, as described above. For lower frequencies, the lack of adjustment may be explained as follows. Below $4 \mathrm{kHz}$, ITD and ILD both provide information about the same spatial dimension (azimuth), but the spatial information provided by low-frequency ILD cues is largely redundant with, and less precise than, the information provided by ITD cues (Knudsen et al., 1994). As a result, once ITD tuning is adjusted in device-reared owls, there is little additional improvement gained in the representation of space by completing the adjustments in ILD tuning. For these reasons, best ILDs for frequencies below $\sim 7 \mathrm{kHz}$ were consistently close to the average acoustic values experienced without the device in both normal and device-reared owls.

In contrast, best ILDs for higher frequencies were, on average, shifted relative to predicted normal values by larger amounts. Because ILDs at these frequencies indicate the elevation of sound sources in barn owls and are not redundant with the spatial information provided by ITDs, these adjustments were able to improve the accuracy of the representation of auditory space in the tectum, even with full adjustment of ITD tuning. However, in most cases these adjustments still fell substantially short of the acoustic effects of the device and thus may have revealed a limit to the capacity of this pathway to modify frequency-specific ILD tuning.

\section{REFERENCES}

Brainard MS, Knudsen EI (1993) Experience-dependent plasticity in the inferior colliculus: a site for visual calibration of the neural representation of auditory space in the barn owl. J Neurosci 13:4589-4608.

Brainard MS, Knudsen EI, Esterly SD (1992) Neural derivation of sound source location: resolution of spatial ambiguities in binaural cues. $\mathrm{J}$ Acoust Soc Am 91:1015-1027.

Carlile S, King AJ (1994) Monaural and binaural spectrum level cues in the ferret: acoustic and the neural representation of auditory space. J Neurophysiol 71:785-801.

Doupe AJ, Konishi M (1991) Song-selective auditory circuits in the vocal control system of the zebra finch. Proc Natl Acad Sci USA 88:11339-11343.

Gold JI, Knudsen EI (1999) Hearing impairment induces frequencyspecific adjustments in auditory spatial tuning in the optic tectum of young owls. J Neurophysiol 82:2197-2209.

Keller CH, Hartung K, Takahashi TT (1998) Head-related transfer functions of the barn owl: measurement and neural responses. Hear Res 118:13-34.

Knudsen EI (1980) Sound localization in birds. In: Comparative studies of hearing in vertebrates (Popper AW, Fay RR, eds), pp 287-322. New York: Springer. 
Knudsen EI (1982) Auditory and visual maps of space in the optic tectum of the owl. J Neurosci 2:1177-1194.

Knudsen EI (1984) Auditory properties of space-tuned units in owl's optic tectum. J Neurophysiol 52:709-723.

Knudsen EI, Esterly SD, du Lac S (1991) Stretched and upside-down maps of auditory space in the optic tectum of blind-reared owls; acoustic basis and behavioral correlates. J Neurosci 11:1727-1747.

Knudsen EI, Esterly SD, Olsen JF (1994) Adaptive plasticity of the auditory space map in the optic tectum of adult and baby barn owls in response to external ear modification. J Neurophysiol 71:79-94.

Margoliash D (1983) Acoustic parameters underlying the responses of song-specific neurons in the white-crowned sparrow. J Neurosci 3:1039-1057.

Margoliash D (1986) Preference for autogenous song by auditory neurons in a song system nucleus of the white-crowned sparrow. J Neurosci 6:1643-1661.

Margoliash D, Fortune ES (1992) Temporal and harmonic combinationsensitive neurons in the zebra finch's HVc. J Neurosci 12:4309-4326.

Mogdans J, Knudsen EI (1992) Adaptive adjustment of unit tuning to sound localization cues in response to monaural occlusion in developing owl optic tectum. J Neurosci 12:3473-3484.

Moiseff A, Konishi M (1981) The owl's interaural pathway is not involved in sound localization. J Comp Physiol 144:299-304.

Olsen JF, Knudsen EI, Esterly SD (1989) Neural maps of interaural time and intensity differences in the optic tectum of the barn owl. J Neurosci 9:2591-2605.
Payne RS (1971) Acoustic location of prey by barn owls (Tyto alba). J Exp Biol 54:535-573.

Perrett DI, Hietanen JK, Oram MW, Benson PJ (1992) Organization and functions of cells responsive to faces in the temporal cortex. Philos Trans R Soc Lond B Biol Sci 335:23-30.

Rolls ET, Baylis GC, Hasselmo ME, Nalwa V (1989) The effect of learning on the face selective responses of neurons in the cortex in the superior temporal sulcus of the monkey. Exp Brain Res 76:153-164.

Rose JE, Gross NB, Geisler CD, Hind JE (1966) Some neural mechanisms in the inferior colliculus of the cat which may be relevant to localization of a sound source. J Neurophysiol 29:288-314.

Takahashi TT, Konishi M (1986) Selectivity for interaural time difference in the owl's midbrain. J Neurosci 6:3413-3422.

Wagner H, Takahashi T, Konishi M (1987) Representation of interaural time difference in the central nucleus of the barn owl's inferior colliculus. J Neurosci 7:3105-3116.

Wise LZ, Irvine DRF (1985) Topographic organization of interaural intensity difference sensitivity in deep layers of cat superior colliculus: implications for auditory spatial representation. J Neurophysiol $54: 185-211$

Yin TCT, Kuwada S (1983) Interaction in low-frequency neurons in inferior colliculus of the cat. III. Effects of changing frequency. J Neurophysiol 50:1020-1042.

Young MP, Yamane S (1992) Sparse population coding of faces in the inferotemporal cortex. Science 256:1327-1331. 